Nadine Beckmann, Stephanie Kadow, Fabian Schumacher, Joachim R. Göthert, Stefanie Kesper, Annette Draeger, Walter J. Schulz-Schaeffer, Jiang Wang, Jan U. Becker, Melanie Kramer, Claudine Kühn, Burkhard Kleuser, Katrin Anne Becker, Erich Gulbins and Alexander Carpinteiro*

\title{
Pathological manifestations of Farber disease in a new mouse model
}

https://doi.org/10.1515/hsz-2018-0170

Received February 27, 2018; accepted May 7, 2018; previously published online June 14, 2018

Abstract: Farber disease (FD) is a rare lysosomal storage disorder resulting from acid ceramidase deficiency and subsequent ceramide accumulation. No treatments are clinically available and affected patients have a severely shortened lifespan. Due to the low incidence, the pathogenesis of FD is still poorly understood. Here, we report a novel acid ceramidase mutant mouse model that enables the study of pathogenic mechanisms of FD and ceramide accumulation. Asaht ${ }^{\text {tmEx }}$ mice were generated by deletion of the acid ceramidase signal peptide sequence. The effects on lysosomal targeting and activity of the enzyme were assessed. Ceramide and sphingomyelin levels were quantified by liquid chromatography tandem-mass spectrometry (LC-MS/MS) and disease manifestations in several organ systems were analyzed by histology and biochemistry. We show that deletion of the signal peptide sequence disrupts lysosomal targeting and enzyme activity, resulting in ceramide and sphingomyelin accumulation. The affected mice fail to thrive and die early. Histiocytic infiltrations were observed in many tissues, as well as lung inflammation, liver fibrosis, muscular disease manifestations and mild kidney injury. Our

\footnotetext{
*Corresponding author: Alexander Carpinteiro, Department of Molecular Biology, University of Duisburg-Essen, Hufelandstraße 55, D-45147 Essen, Germany; and Department of Hematology, University Hospital Essen, Hufelandstraße 55, D-45147 Essen, Germany, e-mail: alexander.carpinteiro@uk-essen.de Nadine Beckmann, Stephanie Kadow, Melanie Kramer, Claudine Kühn and Katrin Anne Becker: Department of Molecular Biology, University of Duisburg-Essen, Hufelandstraße 55, D-45147 Essen, Germany

Fabian Schumacher: Department of Molecular Biology, University of Duisburg-Essen, Hufelandstraße 55, D-45147 Essen, Germany; and Department of Toxicology, Institute of Nutritional Science, University of Potsdam, Arthur-Scheunert-Allee 114-116, D-14558 Nuthetal, Germany

Joachim R. Göthert and Stefanie Kesper: Department of Hematology, University Hospital Essen, Hufelandstraße 55, D-45147 Essen, Germany
}

new mouse model mirrors human FD and thus offers further insights into the pathogenesis of this disease. In the future, it may also facilitate the development of urgently needed therapies.

Keywords: acid ceramidase; ceramide; Farber disease; lysosomal storage disorders.

\section{Introduction}

Farber disease (FD) is a rare lysosomal storage disorder resulting from acid ceramidase (human AC, murine Ac) deficiency (Sugita et al., 1972). AC is encoded by the ASAH1 gene (murine Asah1), located on 8p21.3-p22 (Li et al., 1999). Functionally, AC is a lipid hydrolase and deacylates ceramide to sphingosine. Ceramide is an important lipid mediator and has been implicated in many pathologies, including apoptosis and inflammation (reviewed in Coant et al., 2017; Schuchman and Desnick, 2017). AC deficiency in FD results in an accumulation of lysosomal ceramide (Levade et al., 1995), which is considered the cause of the disease, although the molecular details are unknown.

FD clinically presents with deformed joints, subcutaneous nodules and progressive hoarseness (Moser et al., 2001). It can resemble juvenile idiopathic arthritis

Annette Draeger: Institute of Anatomy, University of Bern, Baltzerstr. 2, CH-3012 Bern, Switzerland

Walter J. Schulz-Schaeffer: Insitute of Neuropathology, University of the Saarland, Kirrberger Str. 100, D-66421 Homburg, Germany

Jiang Wang: Department of Pathology and Laboratory Medicine, UC Health University Hospital, 234 Goodman Street, Cincinnati, $\mathrm{OH}$ 45219, USA

Jan U. Becker: Institute of Pathology, University Hospital Cologne, Kerpener Straße 62, D-50937 Cologne, Germany

Burkhard Kleuser: Department of Toxicology, Institute of Nutritional Science, University of Potsdam, Arthur-Scheunert-Allee 114-116, D-14558 Nuthetal, Germany

Erich Gulbins: Department of Molecular Biology, University of Duisburg-Essen, Hufelandstraße 55, D-45147 Essen, Germany; and Department of Surgery, University of Cincinnati, 231 Albert Sabin Way, ML 0558, Cincinnati, OH 45229, USA 
in infancy, for which it is commonly misdiagnosed at first (Kostik et al., 2013; Sólyom et al., 2014; Torcoletti et al., 2014). FD diagnosis is obtained by demonstration of reduced AC activity and/or abnormally high ceramide levels in cultured cells, biopsy samples or urine (Sugita et al., 1975; Dulaney et al., 1976; Fensom et al., 1979; Kudoh and Wenger, 1982; Antonarakis et al., 1984; Ben-Yoseph et al., 1989; Levade et al., 1996). FD histology is characterized by granulomas with lipid-laden macrophages (Farber et al., 1957). Depending on disease severity and organ involvement, seven subtypes have been classified (Moser et al., 2001). Severely affected patients (subtype I and IV) rarely survive 2 years of age and exhibit lung involvement (type I and IV), neurological deficits (type I) and/or hepatosplenomegaly (type IV). Patients with a less severe phenotype (type II-III, V-VII) may survive into adulthood. So far, the disease variability cannot be explained and the pathophysiology is still poorly understood. Several mutations in different domains of the enzyme have been described, including two single base pair mutations (Gln22His and His23Asp) in the signal peptide sequence (Zhang et al., 2000), but reports on genotype-phenotype correlations are missing for most of the identified ASAH1 mutations.

Currently, no cure for FD exists and treatment options are limited to supportive care. Favorable results on joint manifestations have been reported in response to allogenic hematopoetic stem cell transplantation (Ehlert et al., 2006; Torcoletti et al., 2014) and AC enzyme replacement therapy is currently being developed (He et al., 2017). Unfortunately, both options are unsuitable for patients with pulmonary disease and neurological involvement, which are hallmarks of severe FD (Yeager et al., 2000).

Apart from its role in FD, ceramide accumulation has also been attributed a pathogenic role in a number of other diseases, including for instance, respiratory diseases (i.e. cystic fibrosis; Teichgräber et al., 2008), neurological disorders (i.e. major depression, Gulbins et al., 2013, and neurodegeneration, Filippov et al., 2012), metabolic and cardiovascular disease (reviewed in Iqbal et al., 2017) and (auto-)inflammation [i.e. rheumatoid arthritis (Kosinska et al., 2014) and multiple sclerosis (Singh et al., 1998)].

We have generated an acid ceramidase mutant mouse model to mirror FD. Two further acid ceramidase loss-of-function models exist. The first deleted a large portion of the catalytic domain of acid ceramidase and was reported to be early embryonic lethal (Li et al., 2002). The second model is viable and carries a point-mutation (P361R) outside of the catalytic domain, which is putatively involved in co-factor binding (Alayoubi et al., 2013). By studying disease manifestations in viable models of systemic Ac deficiency, new insights into the pathogenesis of FD and other ceramide-related diseases can be obtained.

\section{Results}

\section{Deletion of acid ceramidase signal peptide disrupts lysosomal targeting and activity of acid ceramidase}

We generated an Asah1 mutant mouse model by flanking Exon1, which encodes the enzyme's signal peptide, with loxP sites and removed the neomycin selection cassette by crossing to Flp recombinant deleter mice. To generate the constitutive model, the floxed mice were mated with EIIacre deleter mice and the resulting heterozygous offspring were subsequently mated among each other, resulting in Wt controls and homozygous Asah1 ${ }^{\text {tmEx }}$ mutants (Figure 1A).

To confirm the deletion of the signal peptide sequence on the RNA level, total RNA was isolated from several tissues, reverse transcribed and subjected to polymerase chain reaction (PCR) using a primer binding to the deleted sequence and a second primer bridging the Exon2/Exon3 junction. Primers directed against the housekeeping protein RPS6 were used as positive controls. As expected, tissues obtained from Asah1 $1^{\text {tmEx }}$ mice show no signal for the Asah1-Exon1 sequence (Figure 1B).

Deletion of Exon1 should result in either a complete knock-out phenotype due to the deletion of the start codon or lead to a mutant protein initiated from an alternative start codon. The later should lack lysosomal targeting because of the deletion of the signal peptide sequence. We studied this by analyzing the intracellular localization of Ac by confocal immunofluorescence analysis. Whereas Ac colocalized with the lysosomal marker protein Lamp1 in Wt cells, no such colocalization was detected in cells derived from Asah1 $1^{\text {tmEx }}$ mutants (Figure 1C). Instead, Ac was distributed diffusely throughout the cytosol in these cells (Figure 1C).

We assessed the effect of disrupted lysosomal targeting on Ac activity in a ceramidase activity assay. We used organ lysates from Asah1 ${ }^{\text {tmEx }}$ mice and NBD-labeled ceramide as the substrate and conducted the experiment at acidic pH. Asah1 ${ }^{\text {tmExx }}$ mutants exhibit reduced ceramidase activity in a tissue-specific manner, with significant reductions in ceramidase activity in liver, spleen, thymus and bone marrow (Figure 1D). Varying degrees of residual activity were observed in all organs (Figure 1D). 

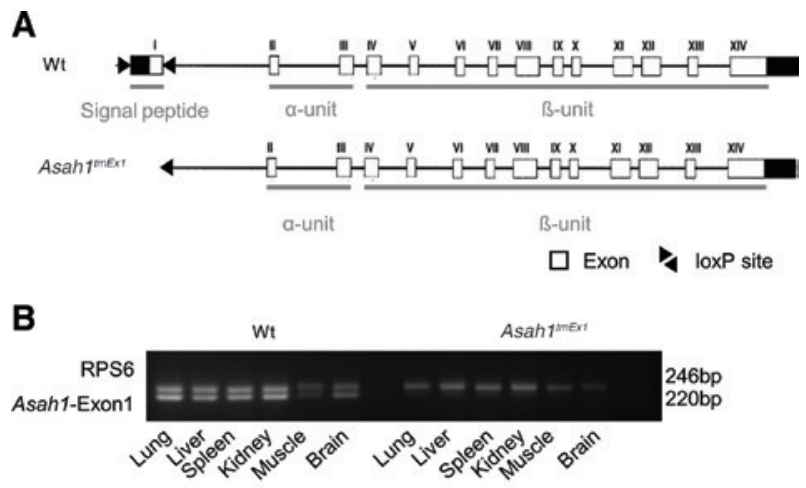

C

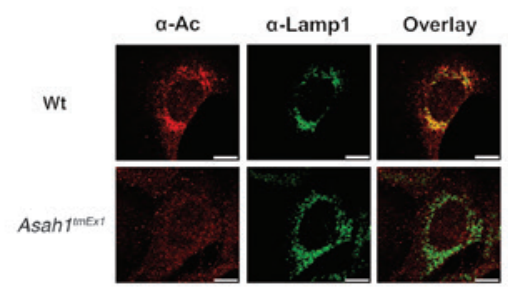

D

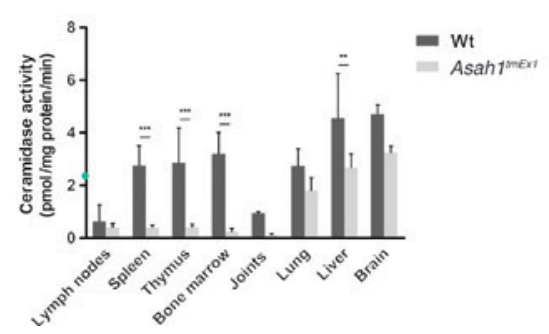

Figure 1: Deletion of acid ceramidase signal peptide disrupts lysosomal targeting and activity of acid ceramidase.

(A) The structure of the murine Asah1 gene in the Asah1 $1^{\mathrm{fl} / \mathrm{fl}}$ Ella-cre ${ }^{\mathrm{tg}}$ constitutive knock-out line is schematically shown. LoxP sites were added flanking Exon1. Upon Ella-cre mediated excision Exon1 is deleted in the acid ceramidase mutant mice (Asah1 ${ }^{\text {tmIExI }}$ ). Image adapted from Moser et al. (2001). (B) Total RNA was isolated from different tissues and reverse transcribed. The absence of Exon1 in Asah $1^{\text {tmEx }}$ mice was analyzed by PCR using a forward primer binding in the targeted region of Exon1. RPS6-PCR was conducted in parallel as a positive control. (C) Immunofluorescence staining of mesenchymal stem cells with antibodies directed against acid ceramidase (Ac, red) and the lysosomal marker lysosomal-associated membrane protein1 (Lamp1, green) to determine cellular localization of Ac in the knock-out model. Representative images of three independent experiments are shown. Scale bar: $10 \mu \mathrm{m}$. (D) Assessment of acid ceramidase activity upon deletion of the signal peptide. Organ lysates were prepared and incubated in the presence of NBD-labeled ceramide. Data are presented as mean $\pm S D(n=4-7$ mice for each group). Multiplicity adjusted $p$-values are indicated (two-way ANOVA with Bonferroni posttests): ${ }^{* \star} p<0.01$; ${ }^{* \star} p<0.001$.

\section{Ceramide and sphingomyelin accumulate in Asah $^{\text {tmExx }}$ mice}

A key feature of FD is the accumulation of ceramide, which is thought to be the cause of the disease. We quantified ceramide levels in several tissues of Asaht $1^{\text {tmEx }}$ mice by liquid chromatography tandem-mass spectrometry (LC-MS/MS). Total ceramide was significantly ele-

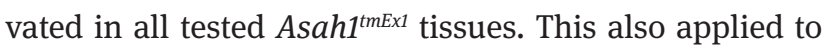
each individual ceramide species tested (Figure 2A-F).

We also tested for potential changes upstream of ceramide by analyzing sphingomyelin levels. We observed an increase of C16- and C24:1-sphingomyelins in the lungs of Asah1 ${ }^{\text {tmEx1 }}$ mice, whereas C18-, C20-, C22-, and C24-sphingomyelins were reduced (Figure $2 \mathrm{G}$ ). In Asah $1^{\text {tmExx }}$ livers, all sphingomyelin species were significantly elevated except for C22- and C24-sphingomyelins (Figure 2H). Similarly, spleens of $A s a h 1^{\text {tmExx }}$ mice had elevated C16-, C22-, C24- and total sphingomyelin levels, but reduced C20-sphingomyelin (Figure 2I) and in Asah1 ${ }^{\text {tmEx }}$ kidneys, all sphingomyelin species were elevated except for C24- and C24-1 sphingomyelin, which were reduced (Figure 2J). In Asah1 $1^{\text {tmEx } 1}$ muscle and brain tissues, all sphingomyelin levels and total sphingomyelin were significantly increased without exception (Figure 2K, L).

\section{Sphingosine, but not sphingosine-1-phos- phate is elevated in Asah1 ${ }^{\text {mEx }}$ mice}

To test if the accumulation of ceramide impacts the activity of other sphingolipid-metabolizing enzymes, we quantified sphingomyelinase activity at acidic $\mathrm{pH}$ and ceramidase activity at neutral $\mathrm{pH}$. Both were not significantly altered in Asaht ${ }^{\text {tmEx }}$ liver samples (Figure 3A, B). We also quantified sphingosine and sphingosine-1-phosphate (S1P) levels in the livers of Asaht ${ }^{\text {mExx }}$ mice. Liver sphingosine levels showed a four-fold elevation in Asaht ${ }^{\text {tmEx }}$ mice (Figure 3C), whereas S1P levels were not significantly different (Figure 3D).

\section{Asah $1^{\text {tmexx }}$ mice fail to thrive, have severely shortened survival and show histopathologi- cal signs of Farber disease}

We tested the biological relevance of the increased ceramide and sphingomyelin levels in the Ac mutant mice by monitoring body weight and survival. Asaht ${ }^{\text {tmEx }}$ mice weighed significantly less than their littermates already at the age of weaning, barely gained weight afterwards and started to lose weight from week 5 onwards (Figure 4A). The earliest death occurred on day 47 and no Asah1 $1^{\text {tmEx }}$ mouse survived for more than 63 days (Figure 4B). No significant differences were seen between male and female Asah1 ${ }^{\text {tmEx }}$ mice regarding weight changes and survival. 
A

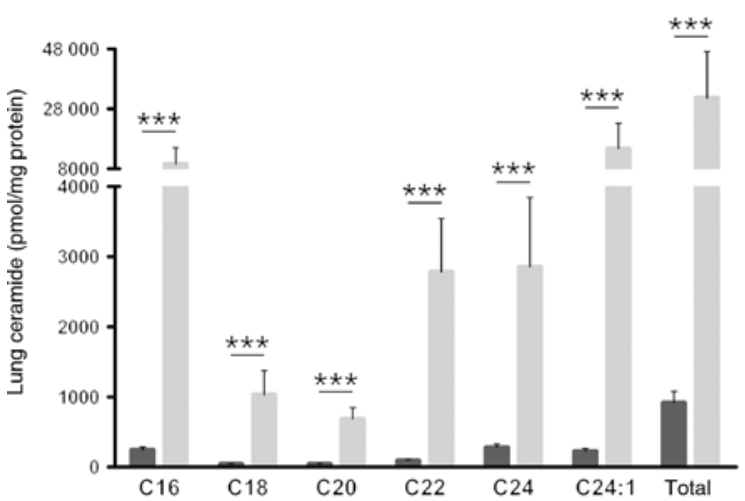

C

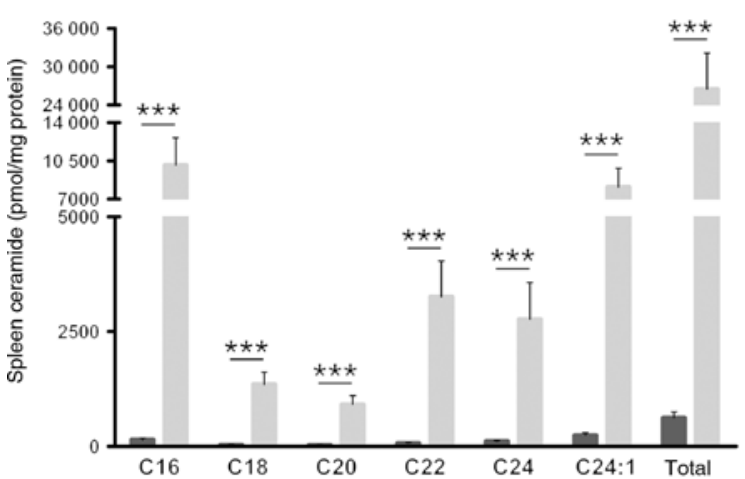

E

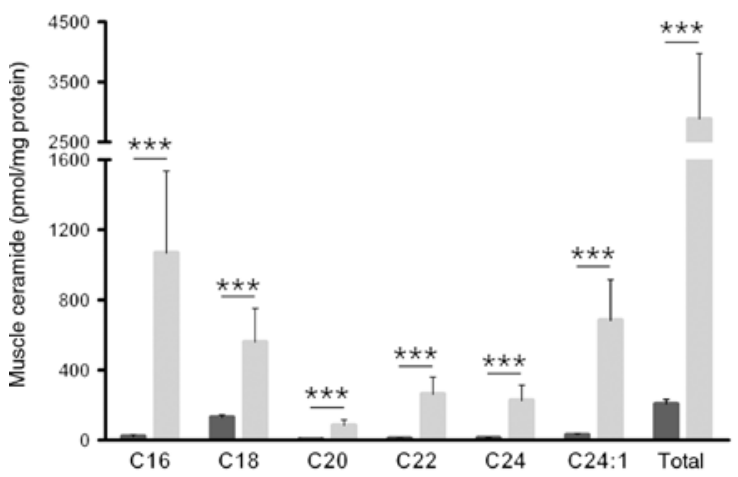

B

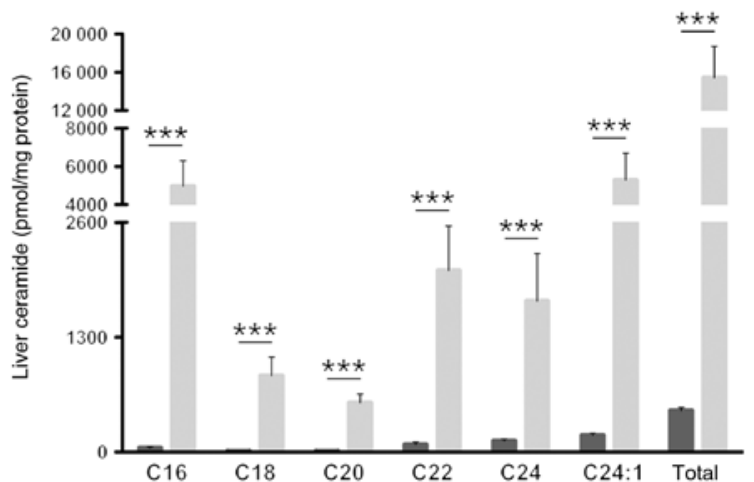

D

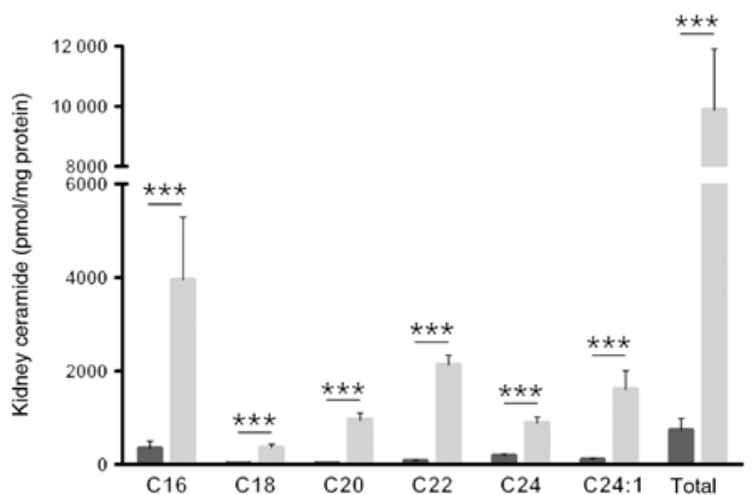

$\mathbf{F}$

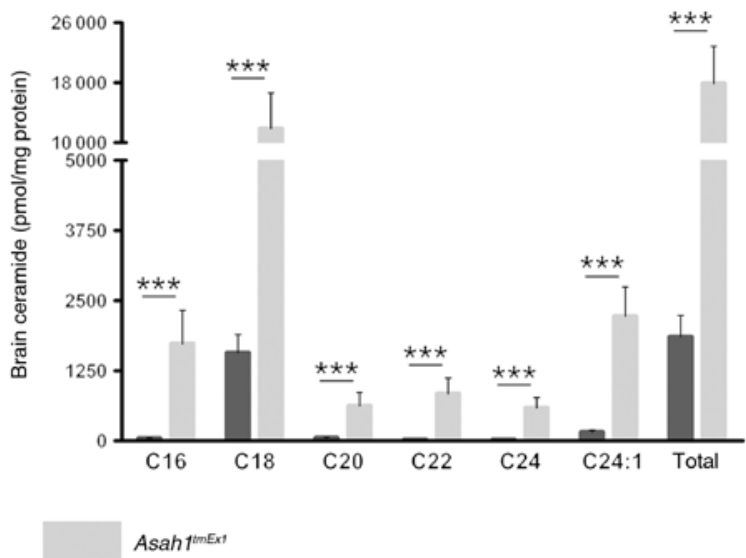

Figure 2: Ceramide and sphingomyelin accumulation in Asah1 ${ }^{\text {tmEx1 }}$ mice.

$(A-L)$ The effect of Asah1 knock-out on ceramide (A-F) and sphingomyelin (G-L) levels were analyzed by rapid resolution LC-MS/MS of snap-frozen organ samples of 6-week-old mice. Data are presented as mean \pm SD $(n=10-13$ mice for each group). Asterisks indicate significant differences of the respective lipid species compared to wildtype (Student's $t$-test): ${ }^{\star} p<0.05 ;{ }^{\star \star} p<0.01 ;{ }^{\star \star \star} p<0.001$.

Lysosomal storage diseases such as FD are characterized by storage bodies (Sikora et al., 2017). In electron-micrographs of liver and brain sections, we identified storage bodies of various shapes and sizes, the most prominent being lamellated, zebra-like bodies in hippocampal sections of the Asah1 ${ }^{\text {tmEx }}$ mutants (Figure 4C).

Clinically, the hallmark feature of FD is joint deformation. We analyzed knee joints of $A s a h 1^{\text {tmExx }}$ mice and noted marked synovial hyperplasia due to the presence of foamy 
G

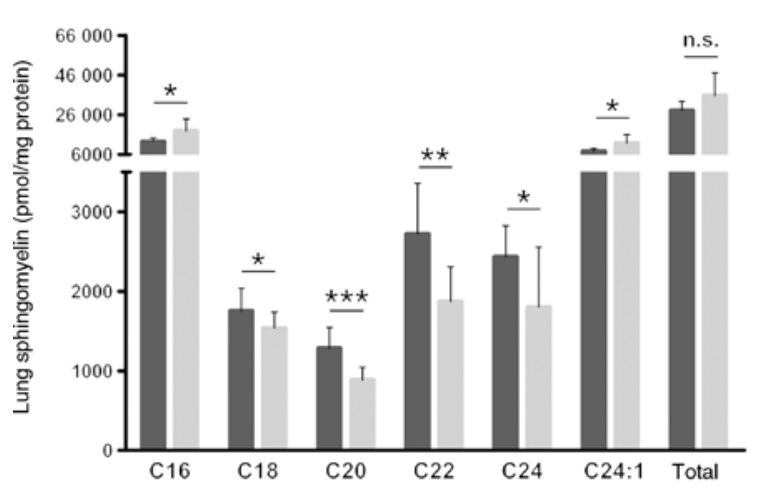

I

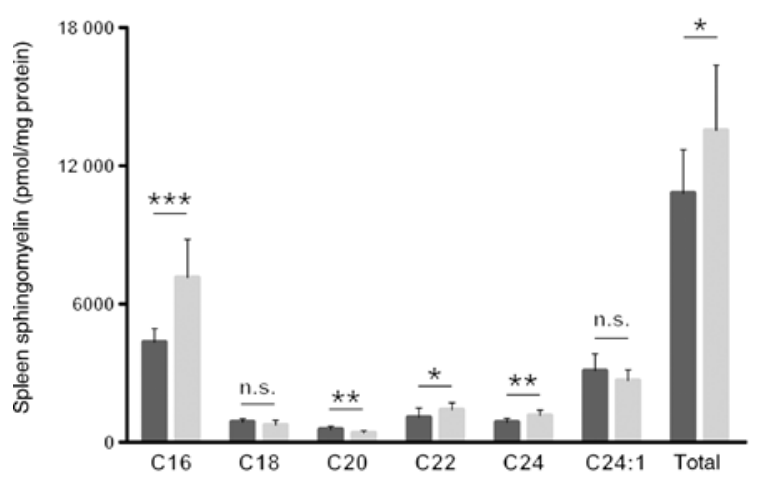

K

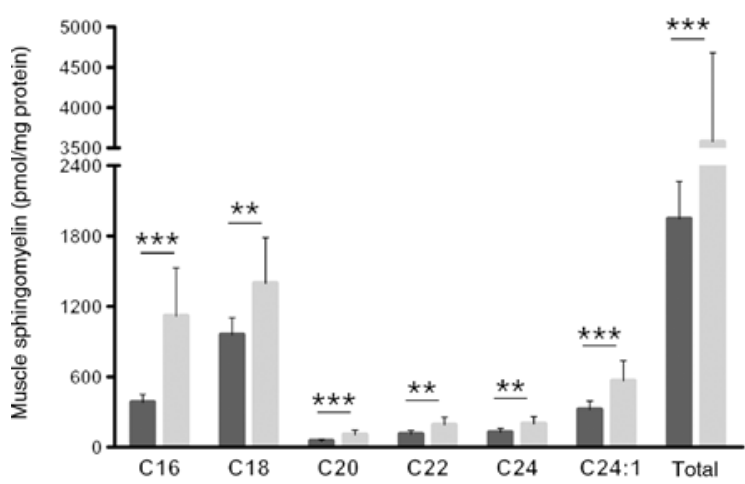

Wt

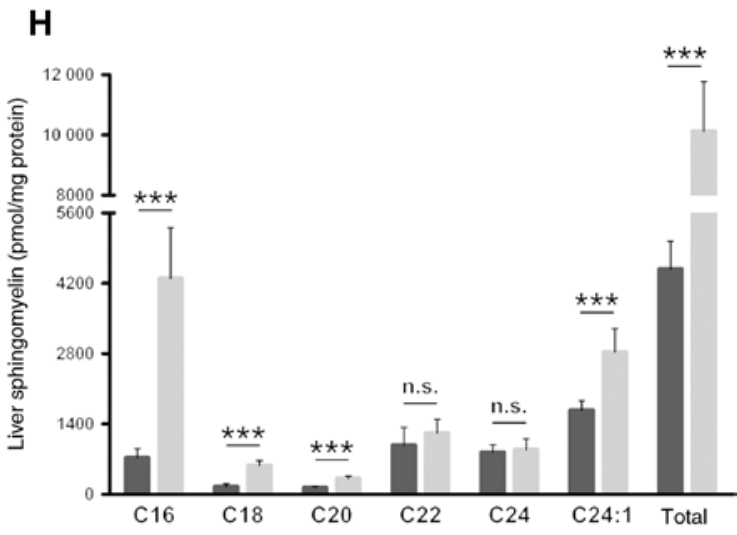

J

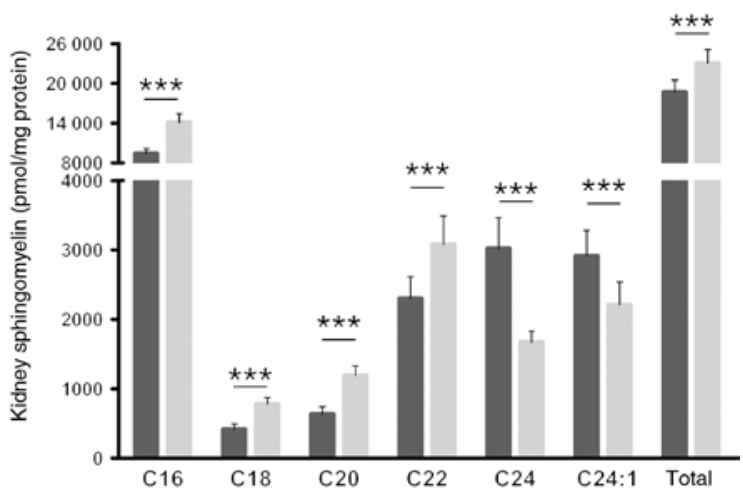

$\mathbf{L}$

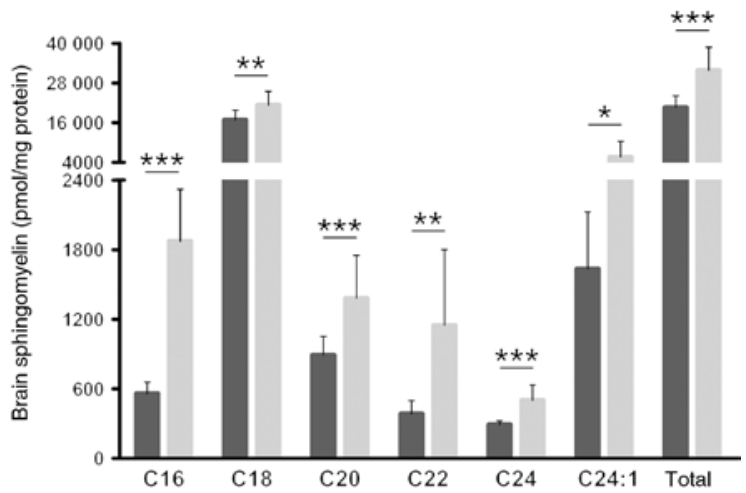

Asah $1^{\text {imexi }}$

Figure 2 (continued)

macrophages (Figure 4D). No other arthritic markers, such as cartilage loss, bone erosion or exudation into the joint cavity were present (Figure 4D).

Granulomas with lipid-laden macrophages are generally considered a key feature of FD and these have been reported in several tissues. Asah1 ${ }^{\text {tmExx }}$ mice also showed such histiocytic infiltrations in the lymphoid organs, which even disrupted the underlying tissue architecture (Figure 4E).

\section{Asah1 ${ }^{\text {tmEx1 }}$ share the characteristic inflamma- tory cytokine profile of FD}

Monocyte chemoattractant protein-1 (MCP-1) has been suggested to be the driver of macrophage infiltration in FD and a characteristic cytokine profile of FD patients has recently been published (Dworski et al., 2017). To test if our mouse model matches this patient cytokine profile, we analyzed the serum levels of MCP-1, MIP-1 alpha, IL-6, 
A
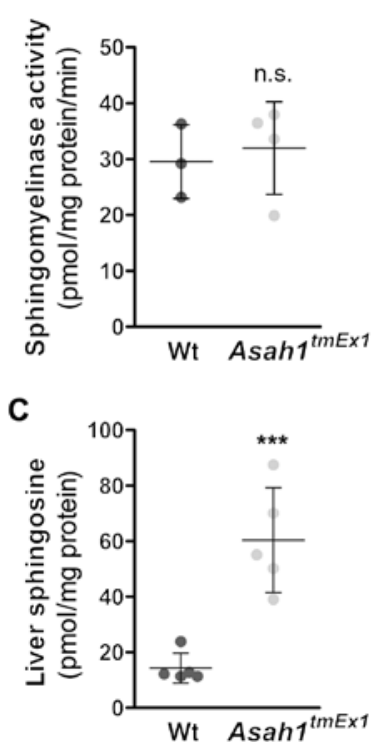

Figure 3: Sphingosine, but not sphingosine-1-phosphate increases in Asaht ${ }^{\text {tmEx } x}$ mice.

(A, B) The effects of Asah1-Exon1 knock-out on the activity on sphingomyelinases was assessed at acidic $\mathrm{pH}(\mathrm{A})$, as well as the effect on the activity on other ceramidases at neutral $\mathrm{pH}$ (B). Lysates were prepared from livers and incubated in the presence of either BODIPY-labeled sphingomyelin (A) or NBD-labeled ceramide (B). Each sample was assessed in duplicate and the mean of each sample is plotted along with the group mean $\pm S D$ ( $n=3-4$ mice for each group). Student's $t$-test showed no significant differences compared to wildtype. (C, D) The effect of Asah1 knock-out on sphingosine (C) and sphingosine-1-phosphate (S1P) (D) levels were analyzed by rapid resolution LC-MS/MS of snap-frozen organ samples of 42/43-day-old mice. Each replicate is presented including mean $\pm S D$ ( $n=5$ mice for each group). Asterisks indicate significant differences compared to wildtype (Student's $t$-test): ${ }^{\star \star \star} p<0.001$.

IL-12, VEGF and IP-10 in our mice. MCP-1, MIP-1 $\alpha$, VEGF and IP-10 were significantly elevated (Figure 5A-D), whereas IL-6 was not significantly different (Supplementary Figure 1) and IL-12 remained below the detection limit of the assay (not shown).

\section{Macrophage accumulation in the central nervous system of Asah $^{\text {tmEx }}{ }^{\text {mice }}$}

Severe FD typically presents with substantial neurological deficits. A recent publication using a mouse model for the disease has provided first insights into the central nervous system (CNS) component of the disease: lipid accumulation due to acid ceramidase deficiency results in storage compounds in the CNS and an accumulation of microglia and/or macrophages, as well as some neurodegeneration late in the disease (Sikora et al., 2017). Given that we also observed storage compounds in the corpus callosum of our mice (Figure 4C), we further evaluated CNS disease manifestations. Luxol-fast blue-reation (LFP-PAS) indicated no obvious demyelination, but showed focal infiltration of white matter tracts by ballooned macrophages (Figure 6A), containing cathepsin D-immunoreactive lysosomes (Figure 6B). These infiltrates were seen in the corpus callosum (Figure 6A), the subcortical white matter of the hemispheres, the corticospinal tract, the optic tract, the cerebellar white matter (Figure 6B), the pyramidal tract and all spinal tracts. Perivascular macrophage cuffs were especially present in the cerebellum and in the meninges. Macrophage accumulation was also present in the olfactory bulb. Meninges showed a scattered, diffuse infiltration by monocytes/macrophages. Neurons of the first two cortical layers exhibited more intense cathepsin D-immunoreactivity compared to controls. The same was observed for plexus choroideus cells. The intraparenchymal macrophage infiltrates were accompanied by astroglial activation, as demonstrated by glial acid fibrillary protein (GFAP) immunoreactivity (Figure 6C).

\section{Hematological disease manifestations in Asah1 ${ }^{\text {tmexx }}$ mice}

Some case reports have indicated hematological manifestations of FD (Antonarakis et al., 1984; Fujiwaki et al., 1992; Mondal et al., 2009), but without any clear consistency. We prepared blood counts to gain further insights and observed a significant reduction of leukocytes in Asah $1^{\text {tmExl }}$ mice (Figure 7A). Upon further differentiation, the number of circulating lymphocytes were reduced, whereas the numbers of granulocytes and monocytes remained unchanged (Figure 7B). The number of erythrocytes was significantly increased (Figure 7C). Whereas the mean corpuscular volume and the average mass of hemoglobin in erythrocytes were significantly reduced (Figure 7D-F), the total amount of hemoglobin did not change (Supplementary Figure 2A). The hematocrit and platelet counts were also normal (Supplementary Figure 2B, C).

Further, we determined weight and cellularity of immune organs relative to the body weight. We performed these and all subsequent hematological analyses with 35-day-old mice, which represents a time-point shortly before the Asah $1^{\text {tmEx }}$ start to manifest signs of severe disease. We found an enlargement of the spleen in Asah $1^{\text {tmEx }}$ mice, while the absolute number of splenocytes was reduced (Figure $7 \mathrm{G}$ ). The size of the thymus was unchanged, but 
A

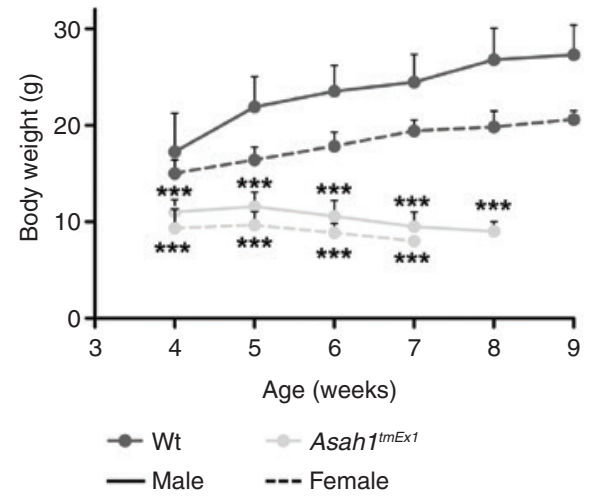

C

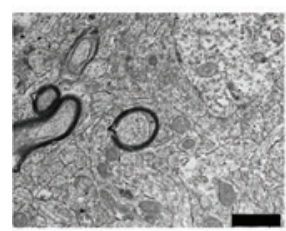

Wt
B

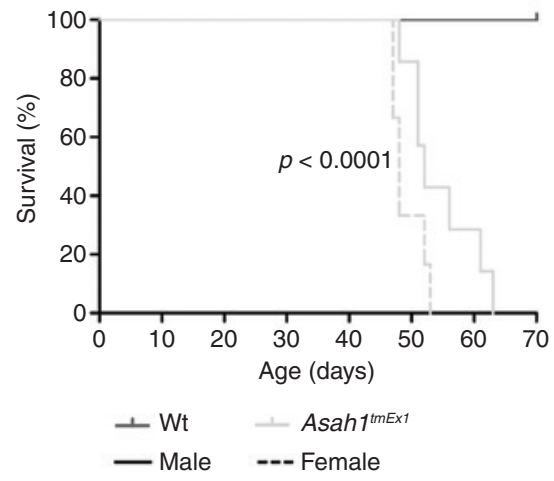

D

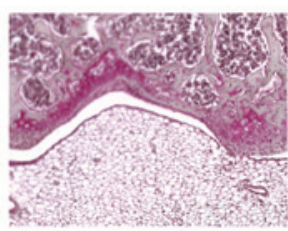

Wt

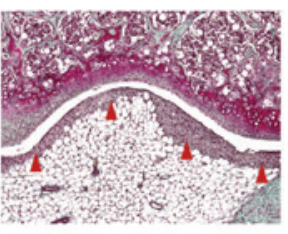

Asah $1^{\text {tmEx1 }}$

E

$\mathrm{Wt}$

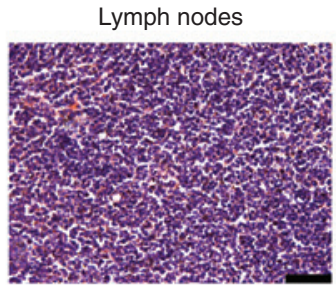

Asaht $1^{\text {tmEx } 1}$

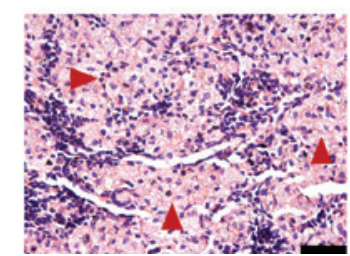

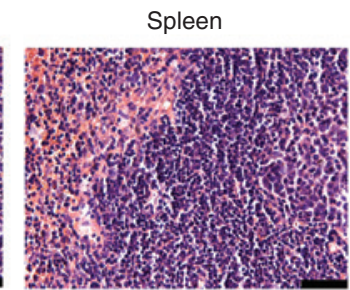
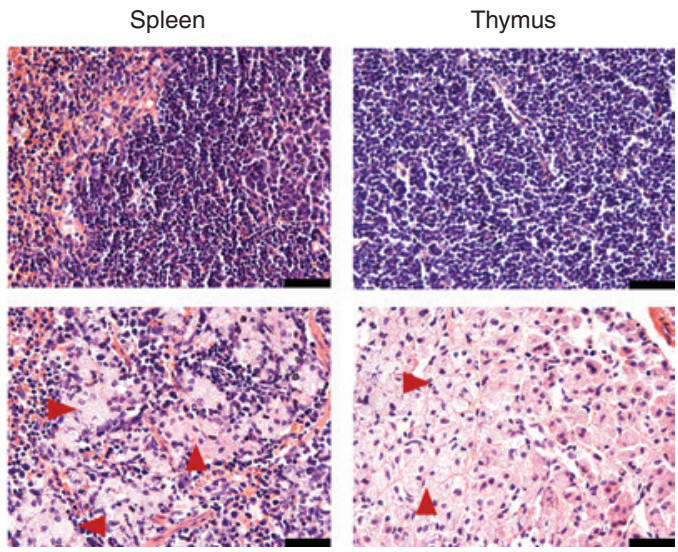

Figure 4: $A s a h 1^{\text {tmEx }}$ mice fail to thrive, severely shortened survival and show histopathological signs of Farber disease.

(A, B) Mice were weighed once a week and the same mice were also monitored daily to assess survival $(n=13$ at the starting point). (A) Body weight data are presented as mean \pm SD Asterisks indicate significant differences compared to the wildtype (Wt) group at the indicated time point (repeated measures ANOVA with Bonferroni posttests): ${ }^{\star \star \star} p<0.001$. (B) Differences in survival were assessed using the log-rank/ Mantel-Cox test and the resulting $p$-values are indicated. The survival of male and female Asah1 ${ }^{\text {tmExx }}$ was not significantly different. (C) Electron micrographs of thin sections through the hippocampi of 6-week-old Wt (left) and Asah $1^{\text {tmEx }}$ mice (right). Zebra-like storage bodies (red circles) lie between myelinated and unmyelinated fibers. Scale bar: $1 \mu \mathrm{m}$. (D, E) histopathology of the effects of ceramide and sphingomyelin accumulation in 6-week-old mice. (D) Safranin0/FastGreen staining and (E) hematoxylin/eosin staining of perfused, paraformaldehydefixed and paraffin-embedded tissue sections. Red arrows indicate foamy macrophage infiltration. Representative images of $\mathrm{n} \geq 6$ mice are shown for each group. Scale bar: $50 \mu \mathrm{m}$.

thymus cellularity was significantly reduced in Asaht ${ }^{\text {tmEx }}$ mice (Figure 7G). A similar pattern was observed for lymph nodes (Figure 7G). In contrast, the bone marrow cellularity was not significantly altered (Figure $7 \mathrm{G}$ ).

Histological analyses revealed that the enlargement of the spleen, lymph node and thymus was due to foamy macrophage infiltration, which disturbed the normal tissue architecture of the immune organs (Figure 4E). We further studied the cellular composition in these organs by flow cytometry. In addition to infiltration with monocytic cells we also observed a significant infiltration with granulocytes (Figure $7 \mathrm{H}$ ). 
A

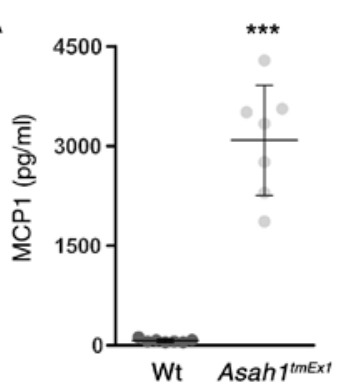

C

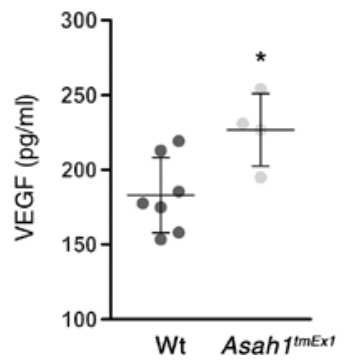

B

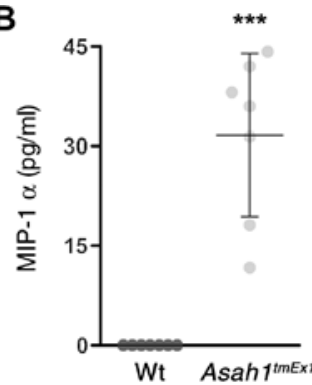

D

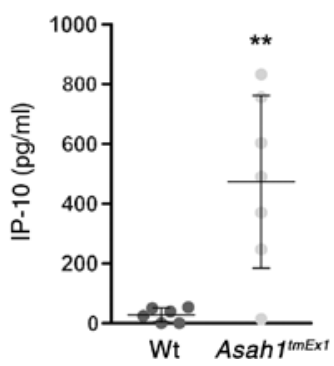

Figure 5: $A s a h 1^{\text {tmExx }}$ share the characteristic inflammatory cytokine profile of FD.

(A-D) Serum cytokine levels were quantified by enzyme-linked immunosorbent assay (ELISA) on day 40-44. Each replicate is presented as well as mean \pm SD. Asterisks indicate significant differences as assess by Student's $t$-test: ${ }^{\star} p<0.05$; ${ }^{\star \star} p<0.01$; $\star \star \star p<0.001$. No significant differences were observed for IL-6 (Supplementary Figure 1) and levels of IL-12 remained below the detection limit.

We also performed more detailed analyses of the lymphocyte subsets in the blood and thymus, as well as of bone marrow progenitor cells. Both B- and T-lymphocytes were significantly decreased in the peripheral blood of Asaht ${ }^{\text {tmEx }}$ mice (Figure 7J). In the thymus, CD4/CD8 double positive $\mathrm{T}$ cells were also markedly reduced, resulting in a marked decrease of CD4 and CD8 single positive T cells as well (Figure $7 \mathrm{~K}$ ). The CD4/CD8 double negative population remained unchanged (Figure $7 \mathrm{~K}$ ).

Analysis of the bone marrow revealed an expansion of the early hematopoietic stem and progenitor cell compartment [lineage negative, Sca1 positive, c-kit positive (LSK) cells] and the lineage committed progenitor cell compartment (c-kit single positive cells), whereas the long-term stem cells (LT-HSC) were unaffected (Figure 7K). The increase in the early progenitor cell compartment did not result in higher blood cell counts (Figure 7A, B, Supplementary Figure 2C).

\section{Lung inflammation in Asah1 ${ }^{\text {tmexx }}$ mice}

Pulmonary involvement has been reported for all severely affected FD patients and over half of the patients for whom
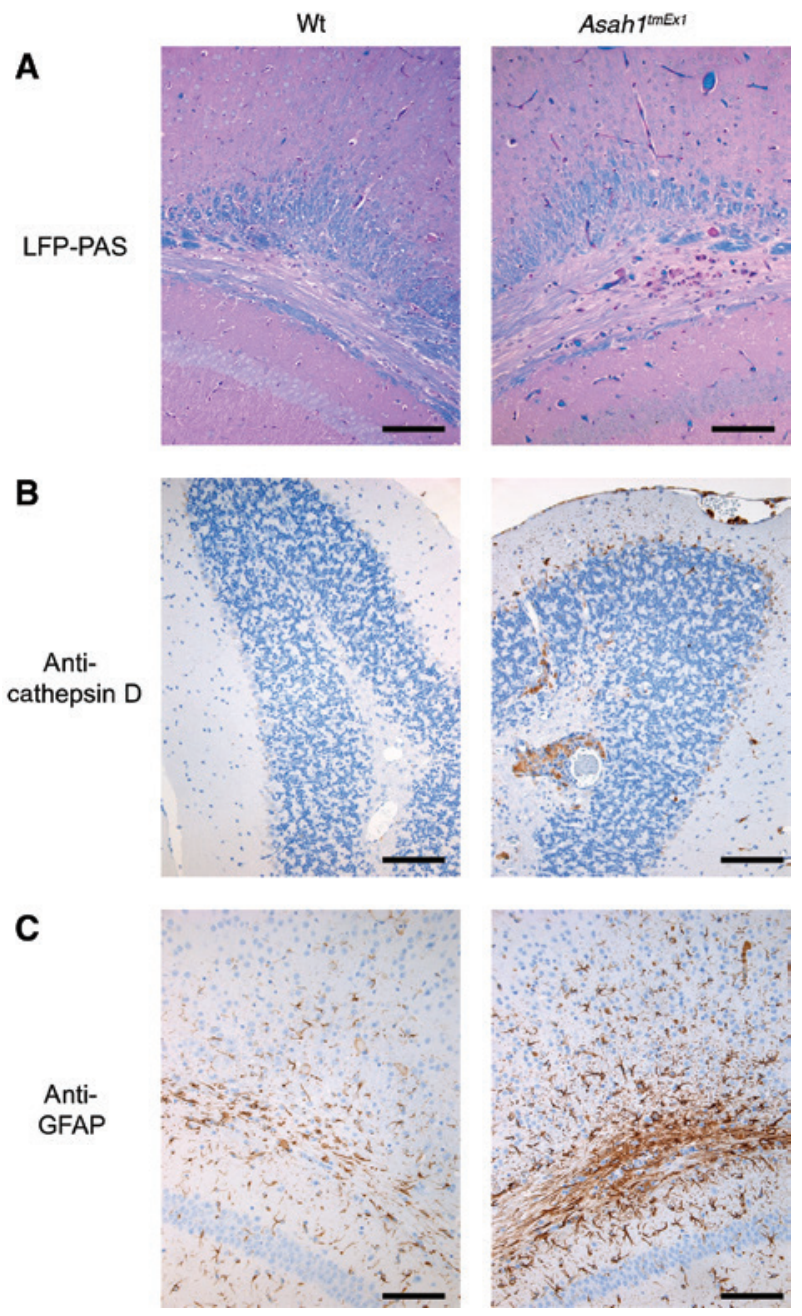

Figure 6: Central nervous system involvement in Asah1 ${ }^{\text {tmExx }}$ mice. (A-C) Paraformaldehyde-fixed and paraffin-embedded brain sections of 5-week-old mice were stained with luxol-fast blue (LFP-PAS) (A), Cathepsin D (B) or glial acid fibrillary protein (GFAP) (C). Representative images of corpus callosum $(A+C)$ and cerebellum $(B)$ are shown for each group. Scale bar: $100 \mu \mathrm{m}$.

a cause of death has been reported have died of pulmonary complications. Additionally, ceramide accumulation is also associated with lung inflammation, cell death and infection susceptibility in cystic fibrosis (Teichgräber

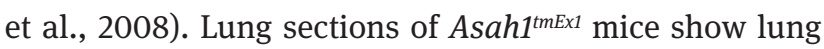
infiltration (Figure 8A), which was subsequently quantified as a significant increase in cell numbers per lung by flow cytometry (Figure 8B). Further characterization showed that this was due to neutrophil infiltration (Figure 8C). Myeloid-derived suppressor cells (MDSCs) were also elevated, but macrophage numbers were not altered (Figure 8C). Dendritic cell- and B-cell numbers decreased significantly (Figure 8D, E), while $\mathrm{T}$ cell numbers also remained unaltered (Figure 8F). 
A

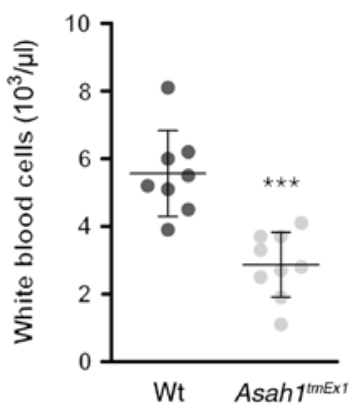

C

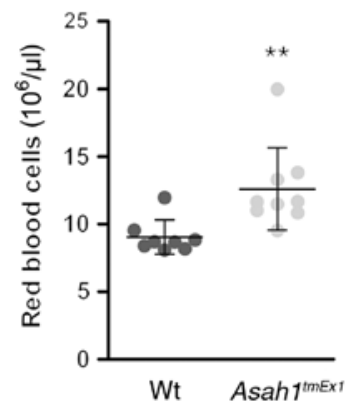

E

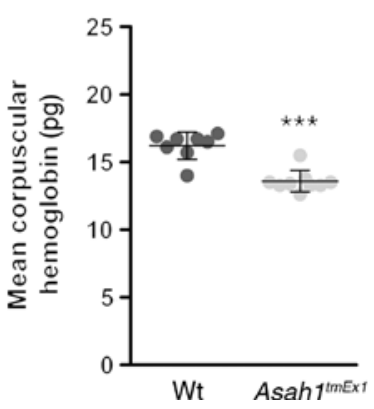

B

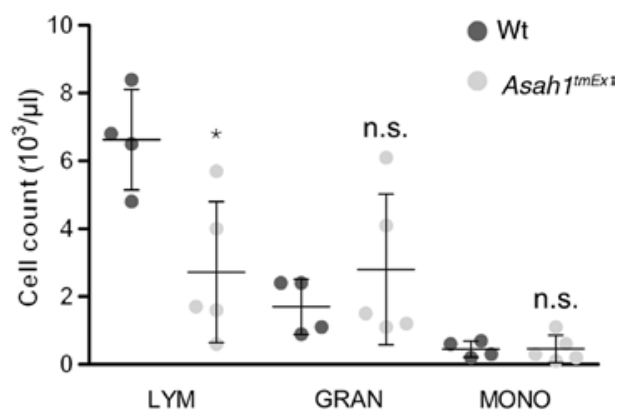

D

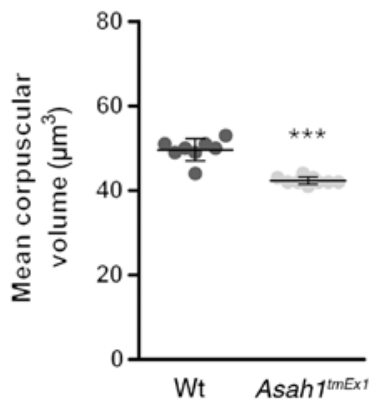

$\mathbf{F}$

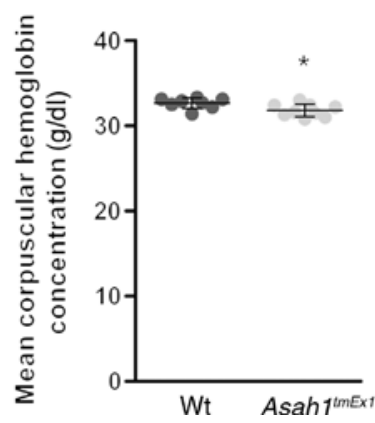

Figure 7: Hematological disease manifestations in Asah1 $1^{\text {tmExi }}$ mice.

(A-F) Hematological manifestations of Farber disease were assessed in 5-6-week-old mice by blood counts in EDTA-anti-coagulated blood using a VetABCTM (Scil). Each replicate is presented including mean $\pm \mathrm{SD}(\mathrm{B})$. Indicated $p$-values are the result of Student's $t$-test: ${ }^{*} p<0.05$; ${ }^{\star \star} p<0.01 ;{ }^{* \star} p<0.001$. No significant differences were observed for hemoglobin, hematocrit and platelets (see Supplementary Figure 2A-C). (G) Organs of 35-day-old mice were resected and weight. Single cell suspensions were prepared and cell numbers determined on a $\mathrm{Z2}$ cell counter (BD, Krefeld, Germany). To correct for the pronounced differences in body weight between the two groups, organ weights are displayed as the ratio of organ weight to body weight and cell counts are displayed as cells per bodyweight. Given are means \pm SD of $\mathrm{n}=4$ mice per group. Indicated $p$-values are the result of Student's $t$-test: ${ }^{\star} p<0.05$; ${ }^{\star \star} p<0.01$; ${ }^{\star \star \star} p<0.001$. (H) Organs of 35 -day-old mice were resected, single cell suspensions were prepared and lymphoid and erythroid cells were excluded via a lineage cocktail containing antibodies against CD3, CD4, CD8, TCRß, CD19, B220, Nk1.1 and Ter119. Granulocytes and monocytes were stained with antibodies directed against Mac- 1 and Ly6G, respectively, and analyzed flow cytometrically. The percentage of mono- or granulocytes per total viable cells is reported. Given are means \pm SD of $n=4$ mice per group. Indicated $p$-values are the result of Student's $t$-test: ${ }^{*} p<0.05 ;{ }^{* \star} p<0.01 ;{ }^{* \star \star} p<0.001$. (I) Blood was collected from 35-day-old mice by cardiac puncture and erythrocytes were lysed in $\mathrm{NH}_{4} \mathrm{Cl}$ hypotonic solution. Leukocytes were subsequently stained with antibodies directed against CD3 and CD19 and analyzed by flow cytometry. Each replicate is presented including mean $\pm S D$ ( $n=4$ mice per group). Indicated $p$-values are the result of Student's $t$-test: ${ }^{\star} p<0.05 ;{ }^{* \star} p<0.01 ;{ }^{* \star *} p<0.001$. (J) The thymi of 35-day-old mice were resected and single cell suspensions were prepared. T cells were stained with antibodies against CD4 and CD8 and analyzed by flow cytometry. Cell numbers are reported as number of cells per bodyweight. Given are means \pm SD of $n=4$ mice per group. Indicated $p$-values are the result of Student's $t$-test: ${ }^{\star} p<0.05$; ${ }^{\star \star} p<0.01$; ${ }^{\star \star \star} p<0.001$. (K) Bone marrow was flushed from both femura of 35 -dayold mice and single cell suspensions were prepared and counted. To exclude mature hematopoetic cells from the analysis, a lineage cocktail with antibodies directed against CD3, CD4, CD8, TCRß, CD19, B220, Nk1.1, CD11b and Gr-1 was used. Hematopoetic stem- and early progenitor cells were then stained with antibodies against c-kit, Sca-1, CD48 and CD150. Cell numbers are reported as number of cells per bodyweight. Given are means $\pm S D$ of $\mathrm{n}=4$ mice per group. Indicated $p$-values are the result of Student's $t$-test: ${ }^{\star} p<0.05 ;{ }^{\star \star} p<0.01 ;{ }^{\star \star \star} p<0.001$. 
G

\begin{tabular}{|c|c|c|c|}
\hline & Wt & Asah $1^{\text {tmext }}$ & $t$-Test \\
\hline \multicolumn{4}{|l|}{ Spleen } \\
\hline Weight [g/g body weight] & $0.576( \pm 0.069)$ & $0.962( \pm 0.211)$ & * \\
\hline Cellularity $\left[10^{6}\right.$ cells $/ g$ body weight] & $8.263( \pm 0.758)$ & $5.723( \pm 2.099)$ & *** \\
\hline \multicolumn{4}{|l|}{ Thymus } \\
\hline Weight $[\mathrm{g} / \mathrm{g}$ body weight] & $0.393( \pm 0.135)$ & $0.402( \pm 0.122)$ & n.s. \\
\hline Cellularity $\left[10^{6}\right.$ cells $/ g$ body weight] & $7.099( \pm 2.604)$ & $0.776( \pm 0.256)$ & $\star \star \star *$ \\
\hline \multicolumn{4}{|l|}{ Lymph node } \\
\hline Weight [g/g body weight] & $0.178( \pm 0.096)$ & $0.131( \pm 0.019)$ & n.s. \\
\hline Cellularity $\left[10^{6}\right.$ cells $/ g$ body weight] & $0.619( \pm 0.280)$ & $0.169( \pm 0.073)$ & ** \\
\hline \multicolumn{4}{|l|}{ Bone marrow } \\
\hline Cellularity $\left[10^{6}\right.$ cells/g body weight] & $2.365( \pm 0.258)$ & $3.212( \pm 0.673)$ & n.s. \\
\hline
\end{tabular}

H

\begin{tabular}{lccc}
\hline & Wt & Asaht $1^{\text {mEx }}$ & $t$-Test \\
\hline Spleen & & & \\
Granulocytes [\% total viable cells] & $2.23( \pm 1.07)$ & $12.50( \pm 2.72)$ & $\star \star \star$ \\
Monocytes [\% total viable cells] & $2.75( \pm 0.59)$ & $6.39( \pm 3.04)$ & $*$ \\
\hline Thymus & & & \\
Granulocytes [\% total viable cells] & $0.28( \pm 0.01)$ & $2.75( \pm 2.04)$ & $\star \star$ \\
Monocytes [\% total viable cells] & $0.05( \pm 0.02)$ & $3.85( \pm 2.55)$ & $\star \star$ \\
\hline Lymph node & & & \\
Granulocytes [\% total viable cells] & $0.11( \pm 0.10)$ & $1.83( \pm 1.08)$ & $\star \star$ \\
Monocytes [\% total viable cells] & $0.57( \pm 0.36)$ & $2.02( \pm 1.06)$ & $*$ \\
\hline
\end{tabular}

I

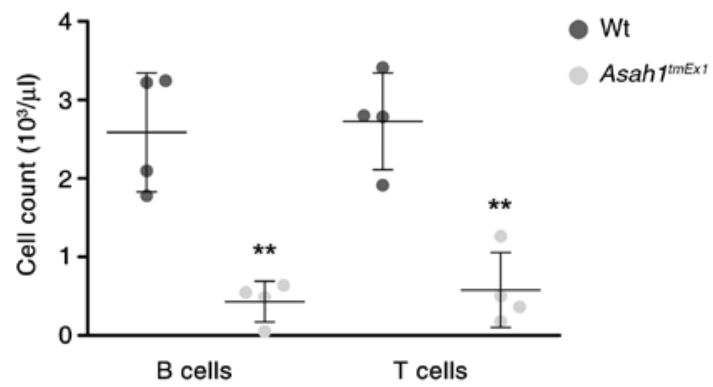

$\mathbf{J}$

\begin{tabular}{lrrr}
\hline & Wt & Asah tmExt $^{\text {t }}$ & $t$-Test \\
\hline Thymic T cell precursors & & & \\
CD4/CD8 double positive $\left[10^{3}\right.$ cells/g body weight] & $4954( \pm 2391)$ & $18( \pm 23)$ & $\star \star$ \\
CD4 single positive $\left[10^{3}\right.$ cells/g body weight $]$ & $972( \pm 127)$ & $177( \pm 107)$ & $\star \star \star$ \\
CD8 single positive $\left[10^{3}\right.$ cells/g body weight $]$ & $381( \pm 53)$ & $186( \pm 66)$ & $\star \star$ \\
CD8 double negative $\left[10^{3}\right.$ cells/g body weight $]$ & $367( \pm 50)$ & $363( \pm 109)$ & n.s. \\
\hline
\end{tabular}

K

\begin{tabular}{|c|c|c|c|}
\hline & Wt & Asah1 1mExt & Test \\
\hline \multicolumn{4}{|l|}{ Early hematopoetic stem and progenitor compartments } \\
\hline c-kit single positive cells $\left[10^{3}\right.$ cells $/ \mathrm{g}$ body weight] & $68.80( \pm 10.60)$ & $154.84( \pm 41.78)$ & ** \\
\hline LSK $\left[10^{3}\right.$ cells/g body weight] & $9.36( \pm 1.07)$ & $12.51( \pm 1.83)$ & * \\
\hline \multicolumn{4}{|l|}{ Long-term stem cells } \\
\hline LT-HSC $\left[10^{3}\right.$ cells/g body weight] & $0.24( \pm 0.03)$ & $0.27( \pm 0.18)$ & n.s. \\
\hline
\end{tabular}

Figure 7 (continued) 
A

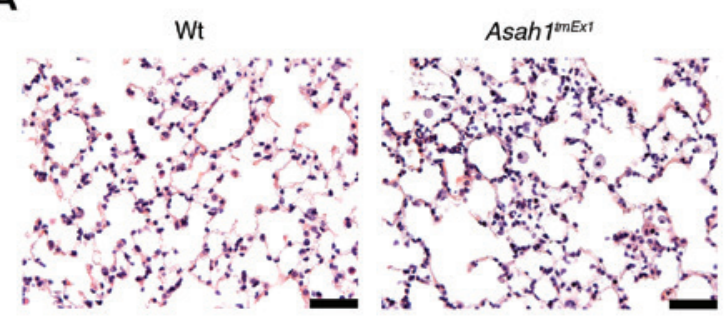

C

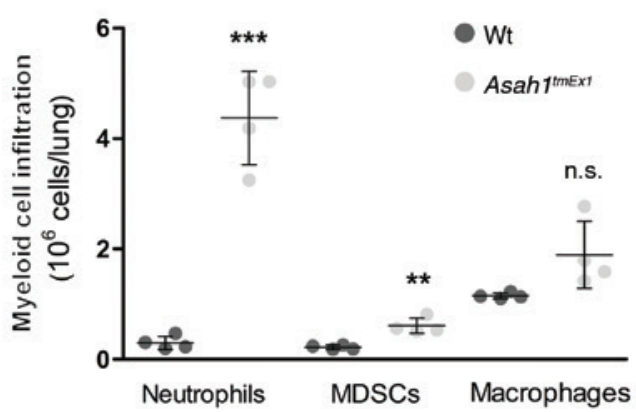

B
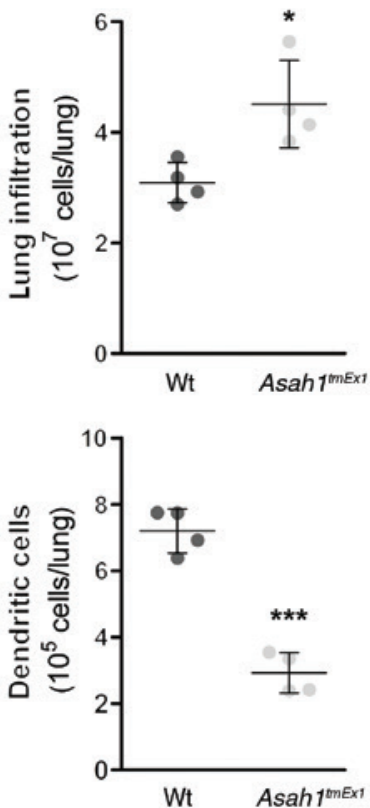

E

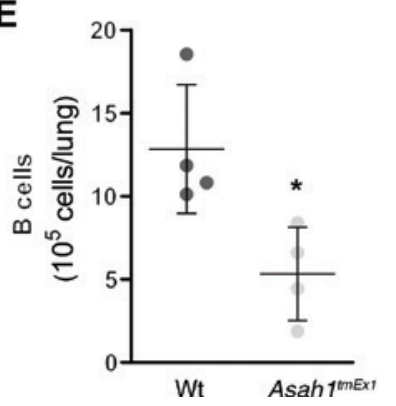

$\mathbf{F}$

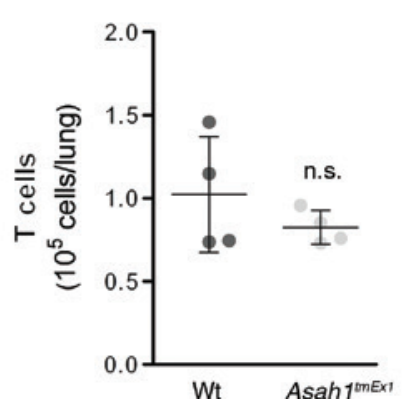

Figure 8: Lung inflammation in $A s a h 1^{\text {tmEx }}$ mice.

(A) Hematoxylin/eosin staining of perfused, paraformaldehyde-fixed and paraffin-embedded lung sections of 6-week-old mice. Representative images of $n \geq 6$ mice are shown for each group. Scale bar: $50 \mu \mathrm{m}$. (B-F) The inflammatory infiltrate in the lung was quantified at 6 weeks and characterized by flow cytometry. (B) Absolute-, (C) myeloid-, (D) dendritic-, (E) T- and (F) B-cell numbers were quantified per lung in $\mathrm{n}=4$ mice. Indicated $p$-values are the result of Student's $t$-test: ${ }^{\star} p<0.05$; ${ }^{\star \star} p<0.01$; ${ }^{\star \star \star} p<0.001$.

\section{Asah1 $^{\text {tmEx1 }}$ mice develop hepatic fibrosis}

Hepatic manifestations have been reported in FD patients and ceramide has been linked to several liver diseases, including hepatic fibrosis (Quillin et al., 2015). Similar to the joints and lymphoid tissues, we observed foamy macrophages in liver sections of $A s a h 1^{\text {tmExl }}$ mice (Figure 9A). This was found to be significant through scoring of the histiocytic infiltration by a blinded investigator (Figure 9B). Signs of early pericentral and pericellular fibrosis were also observed, which we confirmed through fibrosis staining (Figure 9C) and scoring (Figure 9D). We also analyzed clinical parameters that indicate hepatocellular injury. Both glutamate-pyruvate transaminase (GPT) and glutamic-oxaloacetic transaminase (GOT) increased over time and were significantly elevated in 6-week-old
Asah1 ${ }^{\text {tmEx } 1}$ mice (Figure 9E-F). Total serum protein and bilirubin levels, as well as albumin levels were not altered (Supplementary Figure 3C).

\section{Muscular disease manifestations in Asah ${ }^{\text {tmexx }}$ mice}

Despite reports that acid ceramidase expression and activity are very low in skeletal muscle (Li et al., 1998), we observed a significant increase of ceramide in muscle tissue (Figure 2E). Upon observation, Asah1 $1^{\text {tmExI }}$ mice showed some potential indicators of muscular dystrophy (progressive wasting, Figure 4A; scoliosis, waddling gait). Additionally, mutations in the ASAH1 gene have also been reported in a subset of spinal muscular 
A

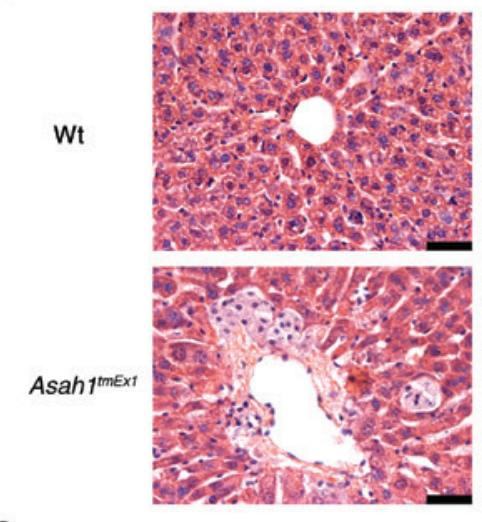

C

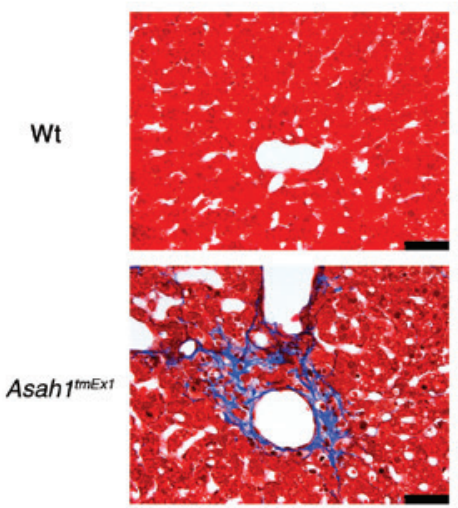

E

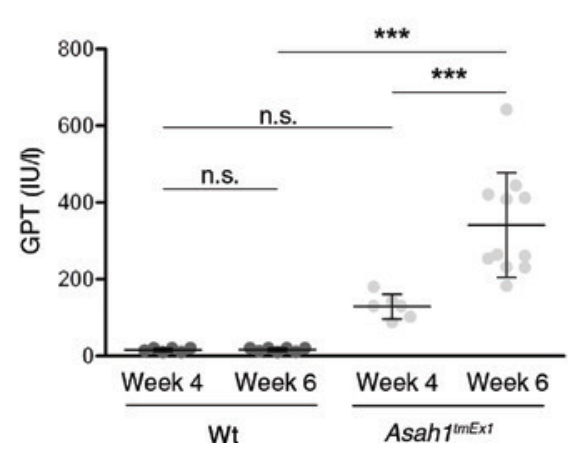

B

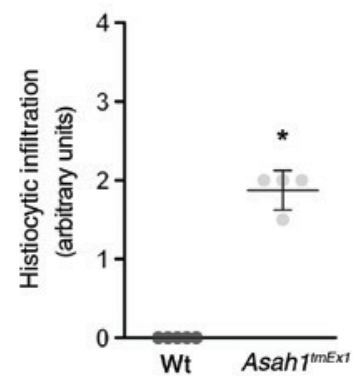

D

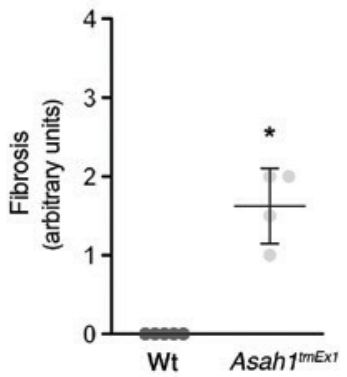

$\mathbf{F}$

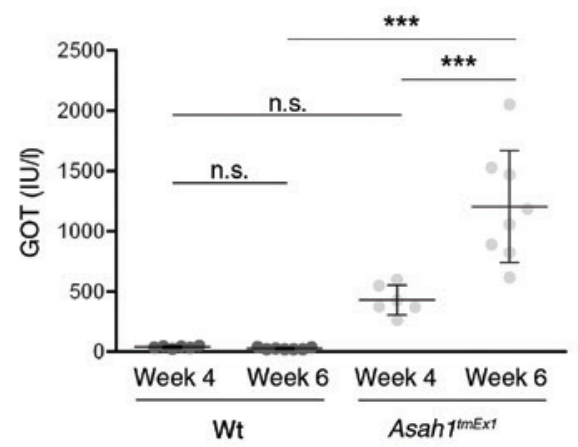

Figure 9: $A s a h 1^{\text {tmExx }}$ mice develop liver fibrosis.

$(A+C)$ Hematoxylin/eosin staining $(A)$ and Masson-Trichrome staining $(C)$ of perfused, paraformaldehyde-fixed and paraffin-embedded liver sections of 6-week-old mice. Representative images of $n>6$ mice are shown for each group. Scale bar: $50 \mu m$. (B+D) Histiocytic infiltration with foamy macrophages (B) and liver fibrosis (D) were scored on a scale of 0 (no signs) to 4 (very severe) by a blinded investigator. Each replicate is presented as well as mean \pm SD. Indicated $p$-values are the result of Wilcoxon signed-rank test: ${ }^{*} p<0.05$. (E-F) Liver parameters were determined in serum using a Spotchem ${ }^{\mathrm{TM}}$ (Scil). Each replicate is presented as well as mean \pm SD. Multiplicity adjusted $p$-values are indicated (two-way ANOVA with Bonferroni posttests): ${ }^{\star \star \star} p<0.001$. No significant differences were detected for total protein, total bilirubin and albumin (see Supplementary Figure 3A-C).

atrophy patients (Zhou et al., 2012; Dyment et al., 2014; Gan et al., 2015; Filosto et al., 2016). This prompted us to check for muscular manifestations of ceramide accumulation in the Asah1 ${ }^{\text {tmEx }}$ mice. Histopathological analysis showed centrally displaced nuclei and some atrophic fibers in 6-week-old Asah1 ${ }^{\text {tmEx1 }}$ mice, which would be consistent with neurogenic atrophy (Figure 10A). We also measured creatinine phosphokinase (CPK)- and lactate dehydrogenase (LDH) levels, two markers for tissue damage. CPK elevations, in particular, indicate damage to tissues with a high ATP-consumption (such as muscle tissue, kidney and brain) and frequently occur in spinal muscular atrophy patients. Both CPK and LDH levels increased significantly over time in 
A
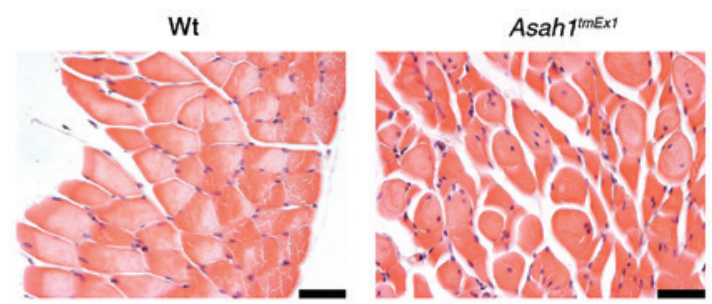

B

C
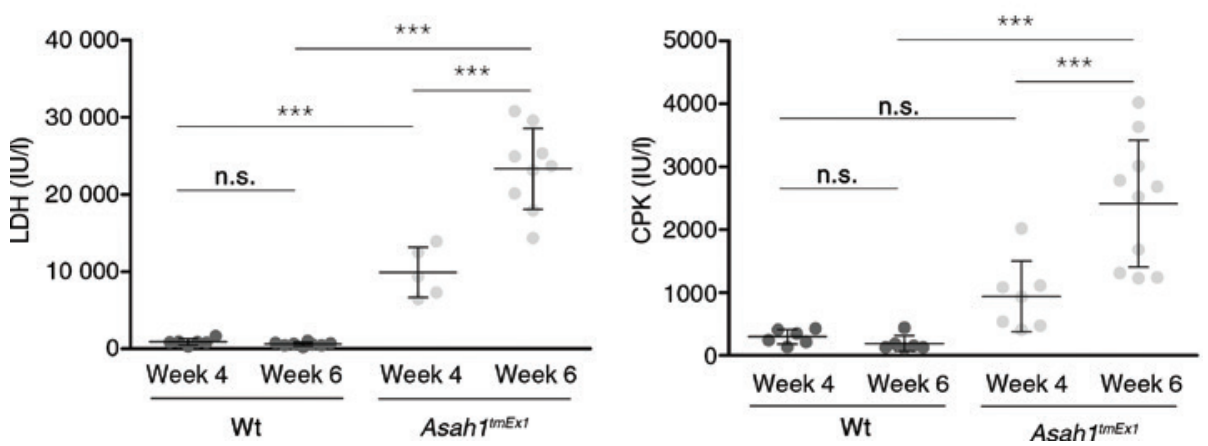

Figure 10: Muscular manifestations in Asah1 ${ }^{\text {tmExx }}$ mice.

(A) Hematoxylin/eosin staining of paraformaldehyde-fixed and paraffin-embedded skeletal muscle (thigh) sections of 6-week-old mice. Representative images of $\mathrm{n}>5$ mice are shown for each group. Scale bar: $50 \mu \mathrm{m}$. (B, C) Serum parameters were determined using a Spotchem ${ }^{\mathrm{TM}}$ (Scil). Each replicate is presented as well as mean \pm SD. Multiplicity adjusted $p$-values are indicated (two-way ANOVA with Bonferroni posttests): ${ }^{\star \star *} p<0.001$.

A
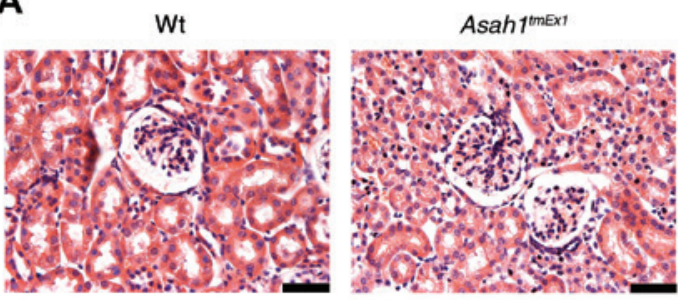

B

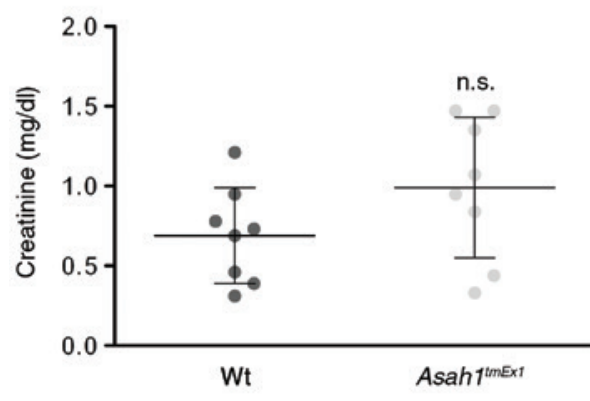

C

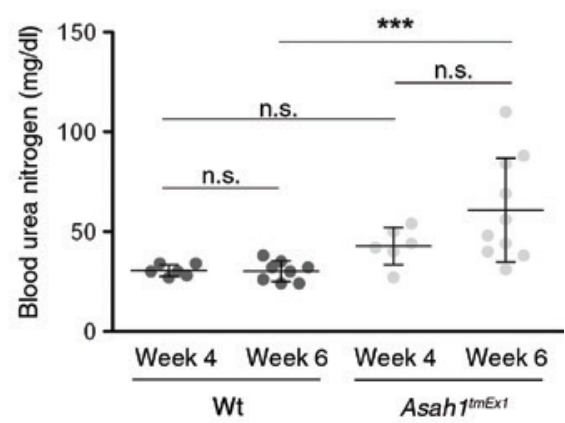

Figure 11: Renal manifestations in Asah1 ${ }^{\text {tmEx } x}$ mice.

(A) Hematoxylin/eosin staining of paraformaldehyde-fixed and paraffin-embedded kidney sections of 6-week-old mice. Representative images of $n>6$ mice are shown for each group. Scale bar: $50 \mu \mathrm{m}$. (B) Serum creatinine was quantified in 6-week-old mice using a colorimetric assay kit. Each replicate is presented as well as mean \pm SD. No significant difference according to Student's $t$-test. (C) Blood urea nitrogen was quantified using a Spotchem ${ }^{\mathrm{TM}}$ (Scil). Each replicate is presented as well as mean \pm SD. Indicated $p$-values are the results of Student's $t$-tests: ${ }^{\star * *} p<0.001$. 
the $A s a h 1^{t m E x x}$ mice, indicating damage to muscle cells (Figure 10B, C).

\section{Renal manifestations in Asah1 ${ }^{\text {tmEx }}$ mice}

Although kidneys are among the organs with the highest expression of AC (Li et al., 1999) and ceramide has been shown to accumulate in the kidneys of FD patients as well (Fujiwaki et al., 1995), little is known about kidney pathologies in FD. We observed no histological abnormalities in Asah $1^{\text {tmEx } x}$ mice (Figure 11A), despite significant ceramide accumulation in Asah1 ${ }^{\text {tmExl }}$ kidneys (Figure 2D). Serum creatinine levels were unaltered in the mutants (Figure 11B), although mutant mice weighed significantly less than their wildtype littermates (Figure 4A). Blood urea nitrogen, generally considered the stronger indicator of renal function, was significantly elevated in 6-week-old Asah1 ${ }^{\text {tmExl }}$ mice (Figure 11C), indicating a decrease of the glomerular filtration rate.

\section{Discussion}

FD is a severe genetic disorder with very limited treatment options. Pathophysiologically, little is known other than that ceramide accumulates due to mutations in the ASAH1 gene. To gain further insights into the disease and to potentially enable the search for new treatment options, we have generated an Asah1 mutant mouse model through EIIa-cre mediated excision of Exon1 (Figure 1A). Two single base pair mutations (Gln22His and His23Asp) in Exon1 were previously identified in FD patients, but the clinical presentation of these patients was not described (Zhang et al., 2000). Exon1 encodes the signal peptide and part of the $\alpha$-unit. Our immunofluorescence analysis shows that rather than a complete knock-out phenotype due to deletion of the start codon, a mutant protein was initiated from an alternative start codon in our model. The mutant protein lacked lysosomal targeting due to the absence of the signal peptide (Figure 1C). Autocatalytic cleavage at acidic $\mathrm{pH}$ has been reported to be an important requirement for Ac activation (Shtraizent et al., 2008). Consequently, we detected reduced Ac activities in all of the tested organs, which were statistically significant in the lymphoid organs and the liver - which were also predominantly affected by FD manifestations. The residual ceramidase activity measured with NBD-ceramide may in part be due to ceramide deacetylation by other enzymes, such as neutral and alkaline ceramidase 1-3, adiponectin receptors (Vasiliauskaite-Brooks et al., 2017) and N-acylethanolamine acid amidase (Tsuboi et al., 2005). The latter enzyme even has high sequence similarity to AC. Further, the residual ceramidase activity in vitro may be due to the truncated, possibly active, mistargeted protein.

Our model appears to closely mimic the human disease and matches another mammalian model of FD - a mouse line with a point mutation in the Asah1 gene, putatively at a site that is involved in the binding of saposin D (Alayoubi et al., 2013). An earlier attempt to knock-out the catalytic domain of Ac abrogated its activity completely and resulted in early embryonic lethality (Li et al., 2002). The authors subsequently showed that Ac expression was upregulated during embryonic genome activation, which was necessary to overcome the ceramide-mediated default apoptosis pathways normally initiated in unfertilized oocytes (Eliyahu et al., 2007). The residual Ac activity in our model is apparently sufficient to lower ceramide levels below this apoptotic threshold during early embryogenesis. Residual ceramide turnover has also been reported for FD patients (Levade et al., 1993).

Disrupted lysosomal targeting and Ac activity in our model resulted in ceramide accumulation as expected for FD (Figure 2A-F). The levels of many sphingomyelin species also increased (Figure $2 \mathrm{G}-\mathrm{L}$ ), but the activity of acid sphingomyelinase was not altered in an in vitro assay (Figure 3A). An increase in sphingomyelins is a new finding in FD, as the levels of other sphingolipids have not been analyzed in detail upon Ac deficiency yet. Only one study has suggested an increase in d18:2 and 24:1 sphingomyelin so far (Jones et al., 2014). Increased sphingomyelin levels may contribute to FD manifestations: NiemannPick diseases type A and B, lysosomal storage disorders caused by acid sphingomyelinase deficiency and resulting in accumulation of sphingomyelin, share some features with FD, including foam cell infiltration, pulmonary and hepatic involvement and central nervous symptoms (reviewed in Schuchman and Desnick, 2017).

We also noted an increase in sphingosine, but not S1P levels in the livers of Asaht ${ }^{\text {tmEx }}$ mice. We chose to analyze livers, because they showed pronounced ceramide alterations (Figure 2B) and pathologies of FD (Figure 9), but without the profound alterations of cellular composition seen in for instance the immune organs (Figure 7) or lungs (Figure 8). The sphingosine increase did not coincide with an increased ceramidase activity at neutral $\mathrm{pH}$ (Figure 3B), suggesting that it is not due to a compensatory effect on neutral ceramidase. Instead, sphingosine accumulation may be the result of enhanced activities of other enzymes with a ceramidase activity, i.e. adiponectin receptors, but further studies are necessary to elucidate which enzyme is 
induced. In any case, the compensatory effect is limited, given that the approximately 50 -fold increase in ceramide gives rise to only a four-fold increase in sphingosine.

Our Asaht ${ }^{\text {tmEx }}$ mouse model mimicked many of the pathologies reported in FD patients: Mice failed to thrive (Figure 4A), survival was severely shortened (Figure 4B), storage bodies were detected by ultrastructural analysis (Figure 4C) and the characteristic foam cell infiltrations were present in several tissues, including the joints (Figure 4D). We also report CNS, lung and liver involvement in our model (Figures 6, 8 and 9), which is typically present in the more severe forms of FD (Moser et al., 2001). Thus, our model seems to best fit FD subtype I and IV. Additionally, our mice also match the recently reported cytokine profile of FD patients (Figure 5) (Dworski et al., 2017).

Given the low incidence rate of $\mathrm{FD}$, comprehensive studies on FD manifestations are difficult and the pathophysiology of the disease is still largely unknown. Using our model, we have analyzed parameters that have previously been implicated in FD case reports, but without any clear consistency. Hematological analysis showed a reduction in blood lymphocytes (Figure 7A, $\mathrm{B}+\mathrm{J}$ ). Red blood cell numbers also increased (Figure 7C), but the erythrocyte indices were reduced (Figure 7D-F). Despite normal hemoglobin levels, these changes may be the consequence of an imbalance of cellular iron distribution in the context of chronic disease. A recent paper suggests that elevations of erythrocytes in Farber mice may be due to poor respiration (Yu et al., 2018). Further studies are required to determine the exact mechanism. The observed leukopenia and normal hemoglobin levels at the first glance contrasts reports from the Asah1 point mutation model, which reported significant elevations of leukocytes in the peripheral blood and increased hemoglobin levels (Alayoubi et al., 2013; Yu et al., 2018). However, it is reasonable that this may reflect varying degrees of disease manifestations in the two models or analysis at different stages of the disease, considering that the point mutation model has a survival time of up to 91 days, whereas no Asah $1^{\text {tmEx }}$ mouse survived for more than 63 days.

We observed a marked reduction in lymphocyte counts in spleen, thymus and lymph nodes relative to the total body weight (Figure 7G). However, the relative organ weights were unchanged for lymph nodes and thymus and even increased for the spleen (Figure 7G). The lymphoid organs were largely infiltrated with granulocytes and monocytic cells (Figure 7H-I). CD4/CD8 double positive- and CD4 and CD8 single positive $\mathrm{T}$ cells (Figure $7 \mathrm{~K}$ ) were significantly decreased in the thymus. These findings indicate a severe defect in T cell maturation in our Farber model. A similar phenotype was also described for the point mutation model
(Dworski et al., 2015). Dworski and colleagues also showed that this $\mathrm{T}$ cell maturation defect aggravates with time and coincides with foam cell infiltration in immune organs, suggesting that the foam cells disrupt the niche for the development of these cells (Dworski et al., 2017). Our findings are in line with these observations.

Similar to the Dworski study, we also observed an increase in immature and committed progenitor cells (Figure 7L). Despite the increase in progenitor cells in the bone marrow, however, the cellular blood counts (Figure 7A, B+J, Supplementary Figure 2C) were not increased. This could be due to an ineffective production in the precursor cell compartment and/or due to an increased consumption of cells. Further experiments, which are beyond the scope of this article, are necessary to gain further insights.

Pulmonary complications are the leading documented cause of death among FD patients. Additionally, ceramide accumulation is known to cause cell death, infection susceptibility and inflammation in cystic fibrosis (Teichgräber et al., 2008). Both prompted us to investigate the lung phenotype of $A s a h 1^{\text {tmExx }}$ mice in more detail. The observed lung inflammation and particularly the neutrophil influx match reports from cystic fibrosis patients and also from the other Farber mouse model (Yu et al., 2018), confirming the pathogenic role of ceramide accumulation in these diseases.

Next to pulmonary complications, liver failure is a major concern in FD and hepatic failure occurs in up to $25 \%$ of FD patients (Bao et al., 2017). Consistent with this, we report hepatocellular injury and liver fibrosis in our model. The later also matches reports from experimental models, indicating that ceramide mediates hepatic fibrosis (Quillin et al., 2015).

ASAH1 mutations have also been reported in a subset of spinal muscular atrophy patients (Zhou et al., 2012; Dyment et al., 2014; Gan et al., 2015; Filosto et al., 2016). We observed some changes in our model that would be consistent with a moto-neuron disease, i.e. signs of neurogenic muscular atrophy (Figure 10A), with biochemical signs of decay of muscular cells (Figure 10B-C) and progressive wasting (Figure 4A). However, further studies are necessary to elucidate this in more detail and to determine the definite cause of CPK elevations in our model, since we cannot exclude damage to other tissues (i.e. heart, brain, kidney) at this time. Nevertheless, our data indicates that the determination of CPK levels in FD patients may turn out to be a useful biomarker for FD. This would help to distinguish FD from juvenile idiopathic arthritis, a disease for which FD is commonly misdiagnosed (Sólyom et al., 2014; Torcoletti et al., 2014). 
So far, little is known about kidney pathologies in FD, which is somewhat surprising considering that kidneys have very high AC expression (Li et al., 1998). Despite the absence of histological abnormalities (Figure 11A), similar serum creatinine levels indicate renal injury when related to the body weight of the mice (Figure 11B), as do elevated blood urea nitrogen levels (Figure 11C). The late onset of these symptoms may indicate that they reflect secondary changes due to the chronic disease in Asah1 ${ }^{\text {tmExx }}$ mice.

Our model not only advances research into FD, but also enables insights into other diseases associated with ceramide accumulation. In the future, it may also facilitate the development of urgently needed therapies in these debilitating diseases.

Further, in our mouse model we flanked Exon-1 of the Asah1 gene with loxP sites. This allows us to generate tissue-specific and inducible knock-outs of Exon1 and consequentially specific deficiency of acid ceramidase. This may become a valuable tool in Farber disease research in the future and impact research into the role of acid ceramidase in various other mechanistic contexts.

\section{Materials and methods}

\section{Animal husbandry}

All mice were maintained on the C57BL/6-J background. Mice were bred and housed in the vivarium of the University Hospital Essen, Germany under pathogen-free conditions as defined by the Federations of European Laboratory Animal Science Associations (FELASA). Mice had ad libitum access to food and water and were kept on a $12 \mathrm{~h} / 12 \mathrm{~h}$ light/dark cycle. Both male and female mice were used for analyses. Breeding of the mice was approved by the State Agency for Nature, Environment and Consumer Protection (LANUV) NRW in Düsseldorf, Germany.

\section{Generation of the Asah $^{\text {tmEx1 }}{ }^{\text {mouse model }}$}

A conditional Asah1 mutant model was generated by GenOway (Lyon, France) through flanking Exon1 with loxP sites and removal of the neomycin selection cassette via crossing to Flp recombinase deleter mice. In detail, the Asah1 cDNA sequence NM_019734 was used to establish the exon/intron organization of the Asah1 gene. On this basis, a targeting vector with two homology regions to the Asah1 genomic sequence in the C57BL/6-J background (one of $5.8 \mathrm{~kb}$ and one of $1.7 \mathrm{~kb}$ ), two loxP sites flanking Asah1 Exon1, a neomycin cassette flanked by FRT sites and a diphtheria toxin A negative-selection marker was constructed. Upon homologous recombination of the targeting vector into $\mathrm{C57BL} / 6$ embryonic stem cells, the resulting chimeric mice were crossbred to $\mathrm{C} 57 \mathrm{BL} / 6$ Flp deleter mice to remove the neomycin cassette. Resulting mice heterozygous for the Neo-excised conditional Asah1 mutant allele were crossed to a C57BL/6 EIIa-cre deleter line and the resulting offspring with the heterozygous excised allele was subsequently mated among each other to obtain homozygous $A s a h 1^{\text {tmExx }}$ mutants and wildtype controls. Genotypes were routinely assessed by PCR using tail biopsies with the following primers:

1. 57447flp: ACAACTGTGTAGGATTCACGCATTCTCC;

2. 57448flp: TCGATCTATGAAATGTCGCTGTCGG;

3. 57445cre: CAGACTAATTCTACCACTTACTGTTCAGACCTC.

Primers 57447flp and 57448flp are combined to detect either the Wt allele (482 bp) or, if present, the floxed allele ( $585 \mathrm{bp}$ ). In a second PCR, primers 57448flp and 57445cre are combined to test for the excision of the floxed allele, yielding a $706 \mathrm{bp}$ fragment if the allele was excised. Thus, Wt mice yield a $482 \mathrm{bp}$ fragment in the first PCR, but no product in the second PCR, whereas Asah1 ${ }^{\text {tmEx }}$ show no product in the first PCR, but yield a 706 bp fragment in the second PCR.

To confirm the deletion of the signal peptide sequence on RNA level, total RNA was isolated from several tissues using the RNeasy Mini kit (Qiagen, Hilden, Germany) and transcribing the RNA to cDNA using the GoScript Reverse Transcription kit (Promega, Mannheim, Germany), both according to the manufacturer's instructions. Subsequently, a PCR was conducted using a forward primer binding to the sequence encoding the signal peptide and a reverse primer bridging the Exon2/Exon3 junction, yielding a $220 \mathrm{bp}$ fragment for the Wt allele or no fragment upon Cre-mediated excision in the Asah1 ${ }^{\text {tmEx }}$ samples. An additional primer pair directed against the housekeeping protein RPS6 was used as a positive control in the same reaction, generating a fragment of $246 \mathrm{bp}$. Primer sequences are as follows:

1. RPS6 fw: ATTCCTGGACTGACAGACAC;

2. RPS6 rev: GTTCTTCTTAGTGCGTTGCT,

3. Asah1 fw: GGGGCCAAAGTCTTCTCACC;

4. Asah1 rev: AAAATCCTCAACGCTGGTGCC.

\section{Intracellular localization of Ac}

Mesenchymal stem cells were generated as described before (Peister et al., 2004) and maintained in IMDM medium (Thermo Fischer Scientific, Waltham, MA, USA) supplemented with $10 \%$ fetal bovine serum (GE Healthcare, Freiburg, Germany) and 10\% horse serum (Thermo Fischer Scientific). Cells were seeded on coverslips and cultured to sub-confluency for $48 \mathrm{~h}$. After washing and fixation with methanol, cells were blocked with 5\% fetal bovine serum in permeabilization/washing solution (BD, Heidelberg, Germany) and subsequently incubated with an antibody directed against the $\beta$-unit of Ac (clone T-20, Santa Cruz Biotechnology, Dallas, TX, USA, $2 \mu \mathrm{g} / \mathrm{ml}$ ), followed by addition of an antibody directed against the lysosomal marker protein Lamp1 (clone 1D4B, Abcam, Cambridge, UK, $1 \mu \mathrm{g} / \mathrm{ml}$ ). After washing with permeabilization/washing solution (BD), cells were stained with Cy3-conjugated donkey-anti-goat IgG (\#705-166$147,7.5 \mu \mathrm{g} / \mathrm{ml}$ ) and AF647-conjugated-anti-rat IgG (\#712-606-150, $7 \mu \mathrm{g} /$ $\mathrm{ml}$; both Jackson ImmunoResearch, Suffolk, UK), washed, mounted with Mowiol (Hoechst, Frankfurt, Germany) and analyzed on a SP5 confocal microscope (Leica microsystems, Wetzlar, Germany).

\section{Determination of ceramidase and sphingomyelinase activity}

Mice were sacrificed by $\mathrm{CO}_{2}$ exposure. Bone marrow was flushed from both femurs and tibias with PBS, pelleted by centrifugation and 
snap-frozen. All other indicated organs were harvested, snap-frozen and pulverized under liquid nitrogen using a mortar and pestle. All samples were lysed in $250 \mathrm{~mm}$ sodium acetate, 1\% NP-40 in a TissueLyser (Qiagen, Hilden, Germany) using $5 \mathrm{~mm}$ stainless steel beads (Qiagen) for $5 \mathrm{~min}$ at $50 \mathrm{~Hz}$. An aliquot was removed and diluted to $0.1 \%$ NP-40 for protein determination using the Bradford protein reagent (BioRad, München, Germany). The NBD-labeled ceramide(Cayman Biochemical) or BODIPY-labeled sphingomyelin substrates (ThermoFisher Scientific) were dissolved in assay buffer $(250 \mathrm{~mm}$ sodium acetate, $0.1 \% \mathrm{NP}-40$ ) and sonicated in an ultrasonic bath for $10 \mathrm{~min}$ to induce micelle formation. Samples were sonicated separately in parallel to the substrate. The substrate solution was then added to the samples to a final concentration of $180 \mathrm{pmol}$ substrate/ sample in $250 \mathrm{~mm}$ sodium acetate, $0.1 \% \mathrm{NP}-40$ at either pH 4.5 (acid ceramidase activity), pH 5.0 (acid sphingomyelinase activity) or $\mathrm{pH}$ 7.6 (neutral ceramidase activity). The reaction mixtures were incubated for $1-2 \mathrm{~h}$ at $37^{\circ} \mathrm{C}$ with $300 \mathrm{rpm}$. The reaction was terminated by lipid extraction with chloroform:methanol (2:1, v/v). Phases were separated by centrifugation for $5 \mathrm{~min}$ at $15000 \mathrm{~g}$ and the organic phase was collected dried in a SpeedVac at $37^{\circ} \mathrm{C}$. Dried lipids were resuspended in chloroform:methanol $(2: 1, \mathrm{v} / \mathrm{v})$ and spotted onto a thin layer chromatography plate (Macherey-Nagel, Düren, Germany). Thin layer chromatography was conducted with ethyl acetate:acetic acid (100:1, v/v, ceramidase assays) or chloroform:methanol (80:20, $\mathrm{v} / \mathrm{v}$, sphingomyelinase assays) as running buffer. The dried plates were imaged using a Typhoon FLA 9500 (GE) and spots were quantified with ImageQuant (GE Healthcare).

\section{Ceramide and sphingomyelin quantification}

Ceramides and sphingomyelin were extracted from indicated tissues and quantified as recently described (Huston et al., 2016). Briefly, lipid extraction was performed using C17-ceramide and C16-d31-sphingomyelin as internal standards. Sample analysis was carried out by rapid-resolution LC-MS/MS using a Q-TOF 6530 mass spectrometer (Agilent Technologies, Waldbronn, Germany) operating in the positive ESI mode. The precursor ions of ceramides [C16-ceramide $(\mathrm{m} / \mathrm{z}$ 520.508), C17-ceramide $(\mathrm{m} / z$ 534.524), C18-ceramide $(\mathrm{m} / z$ 548.540), C20-ceramide $(\mathrm{m} / \mathrm{z}$ 576.571), C22-ceramide $(\mathrm{m} / z$ 604.602), C24-ceramide $(\mathrm{m} / z$ 632.634) and C24:1-ceramide $(\mathrm{m} / z$ 630.618)] were cleaved into the fragment ion $m / z 264.270$. The precursor ions of sphingomyelin [C16-sphingomyelin $(\mathrm{m} / z$ 703.575), C16-d31-sphingomyelin $(\mathrm{m} / \mathrm{z}$ 734.762), C18-sphingomyelin ( $\mathrm{m} / z$ 731.606), C20-sphingomyelin $(\mathrm{m} / \mathrm{z}$ 759.638), C22-sphingomyelin ( $\mathrm{m} / z$ 787.669), C24-sphingomyelin $(\mathrm{m} / z$ 815.700) and C24:1-sphingomyelin $(m / z$ 813.684)] were cleaved into the fragment ion $m / z$ 184.074. Quantification was performed with MassHunter Software (Agilent Technologies). Determined sphingolipid amounts were normalized to the actual protein content, as assessed by Bradford assay, of the tissue homogenate used for extraction.

\section{Electron microscopy analysis}

Samples were cut to tissue blocks of approx. 3-6 mm, fixed and processed for semi-thin sectioning and ultrastructural analysis by electron microscopy. All samples were fixed in $2.5 \%$ glutaraldehyde in sodium cacodylate buffer ( $\mathrm{pH}$ 7.4) and embedded in epoxy resin as previously described (Zaugg et al., 1999). Ultrathin sections were viewed with a Philips CM 12 transmission electron microscope and micrographs obtained using a Morada camera (12 Megapixel) with AnalySIS ITEM software.

\section{Histopathological assessment}

Mice were sacrificed by $\mathrm{CO}_{2}$ exposure and perfused with $0.9 \%$ sodium chloride followed by $4 \%$ paraformaldehyde (PFA) via the right ventricle. Organs were dissected and fixed further in 4\% PFA, dehydrated with ethanol, embedded in paraffin and trimmed to 4-6 $\mu \mathrm{m}$ thin sections (peripheral organs) or $1-5 \mu \mathrm{m}$ thin sections (brain). These were stained as indicated with hematoxylin and eosin, SafraninO/FastGreen, Luxol blue/PAS or Masson Trichrome staining kit (Sigma-Aldrich, St Louis MO, USA). For immunhistochemical analyses, the following antibodies were used: polyclonal rabbit anti-GFAP (\#Z0334, Dako, Glostrup, Denmark) and anti-Cathepsin D (\#1029, R\&D Systems, Minneapolis MN, USA). Slides were analyzed on a Leica SP5 (peripheral organs) or on an Olympus BX40 (brain). Histiocytic infiltration in various tissues and liver fibrosis were scored on a scale of 0 (no signs) to 4 (very severe) by a blinded investigator.

\section{Cytokine analysis}

Cytokine levels were quantified by enzyme-linked immunosorbent assay. Mice were sacrificed by $\mathrm{CO}_{2}$ exposure, blood was drawn from the V. cava inferior and serum was obtained by centrifugation at 2500 $g$ for 20 min. MCP-1, MIP-1, IL-6, IL-12 and VEGF levels were quantified using R\&D Quantikine ELISA kits (R\&D Systems, Minneapolis, MN, USA), CXCL1 levels by using the respective Abcam ELISA kit (Abcam, Cambridge, UK), according to manufacturer's instructions.

\section{Blood counts and clinical chemistry analysis}

Blood was drawn and anti-coagulated with EDTA for blood counts using a VetABC ${ }^{\text {тм }}$ (Scil). For differential blood counts, blood smears from the EDTA-anti-coagulated blood were prepared and two smears per mouse were counted manually.

Serum was obtained by centrifugation at $2500 \mathrm{~g}$ from coagulated blood samples and analyzed using a SpotChem ${ }^{\mathrm{TM}}$ EZ chemistry analyzer with the corresponding parameter strips (Scil, Viernheim, Germany). When necessary, samples were diluted to enable measurement of small sample volumes or when samples exceeded the maximum detection limit of a given parameter. Serum creatinine levels were quantified using a colorimetric assay kit (Cayman, Ann Arbor, USA).

\section{Immune organ weights and cellularity}

Spleen, thymus and inguinal lymph nodes were resected and weighed. For single cell suspension organs were passed through a $70 \mu \mathrm{M}$ cell strainer (BD, Heidelberg, Germany). Organ cellularity was determined on a Z2 cell counter (Beckmann Coulter, Krefeld, Germany). 
Table 1: Antibodies for flow cytometrical analysis.

\begin{tabular}{llllr}
\hline Antigen & Label & Clone & Supplier & Concentration $(\boldsymbol{\mu g} / \mathrm{ml})$ \\
\hline B220 & PerCP-Cy5.5 & RA3-6B2 & BioLegend \\
CD11b & PE-Cy7 & M1/70 & eBioscience \\
CD11b & PerCP-Cy5.5 & M1/70 & BD Pharmingen & 0.5 \\
CD150 & APC & TC15-12F12.2 & BioLegend & 1 \\
CD19 & PerCP-Cy5.5 & 1D3 & BD Pharmingen & 4 \\
CD3 & PerCP-Cy5.5 & 145-2C11 & eBioscience & 2 \\
CD4 & PE-CY7 & RM4-5 & BD Pharmingen & 1 \\
CD4 & PerCP-Cy5.5 & RM4-5 & BD Pharmingen & 4 \\
CD48 & PE & HM-48-1 & BD Pharmingen & 1 \\
CD8 & APC-Cy7 & $53-6.7$ & eBioscience & 4 \\
CD8 & PerCP-Cy5.5 & $53-6.7$ & BioLegend & 2 \\
C-kit & APC-Cy7 & ACK2 & eBioscience & 4 \\
Gr-1 & PerCP-Cy5.5 & RB6-8C5 & BD Pharmingen & 0.5 \\
Ly-6G & FITC & 1A8 & BD Pharmingen & 5 \\
Nk1.1 & PerCP-Cy5.5 & PK136 & eBioscience & 2 \\
Sca-1 & PE-Cy7 & D7 & BD Pharmingen & 2 \\
TCR beta & PerCP-Cy5.5 & H57-597 & eBioscience & 2 \\
Ter119 & PerCP-Cy5.5 & TER119 & eBioscience & \\
\hline
\end{tabular}

\section{Flow cytometry analysis of immune organs}

Organs were dissected, single cell suspensions were prepared, stained with fluorochrome-conjugated antibodies and analyzed by flow cytometry on a LSRII flow cytometer (BD, Heidelberg, Germany). To distinguish mature hematopoietic cells from stem- and progenitor cells in the bone marrow, bone marrow cells were stained with a lineage cocktail containing antibodies against the following antigens: CD3, CD4, CD8, TCR $\beta$, CD19, B220, Nk1.1, CD11b and Gr-1. Hematopoietic stem- and progenitor cells were stained with antibodies directed against c-kit, Sca-1, CD48 and CD150.

For the analysis of granulomonocytic cells, a lineage cocktail containing antibodies against CD3, CD4, CD8, TCR $\beta$, CD19, B220, Nk1.1 and Ter119 was used to distinguish lymphoid and erythroid cells. Granulocytes and macrophages were stained with antibodies directed against CD11b and Ly6-G.

T-cells in the thymus we stained with anti-CD4 and anti-CD8 antibodies.

Information regarding antibody concentrations, fluorochromelabels and manufacturers are summarized in Table 1.

\section{Characterization of lung inflammatory infiltrate}

Immune cell infiltration into the lung was characterized and quantified by flow cytometry. Absolute cell counts were obtained from the live cell gate using forward- and sideward scatter. Specific cell types were defined as follows: Neutrophils: CD11b ${ }^{+}, \mathrm{Ly}^{6} \mathrm{G}^{+}, \mathrm{F} 4 / 80^{-}$ ; Macrophages: CD11b+ $\mathrm{Ly}^{+} \mathrm{G}^{-}, \mathrm{F} 4 / 80^{+}$; MDSCs: CD11b+ $\mathrm{Ly}^{+} \mathrm{G}^{+}$,

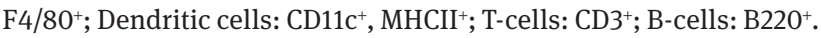
The analysis was conducted on a FACS calibur (BD, Heidelberg, Germany).

Information regarding antibody concentrations, fluorochromelabels and manufacturers are summarized in Table 2.
Table 2: Antibodies for characterization of the lung inflammatory infiltrate.

\begin{tabular}{llllr}
\hline Antigen & Label & Clone & Supplier & $\begin{array}{r}\text { Concentration } \\
(\mu \mathrm{g} / \mathrm{ml})\end{array}$ \\
\hline B220 & FITC & RA3-6B2 & BioLegend & 0.5 \\
CD11b & PE & M1/70 & BioLegend & 0.2 \\
CD11C & PE & N418 & eBioscience & 0.5 \\
CD3 & PerCP-Cy5.5 & $145-2 C 11$ & eBioscience & 2 \\
Ly-6G & APC & 1A8 & BioLegend & 0.2 \\
F4/80 & Alexa488 & BM8 & BioLegend & 1 \\
MHC-II & APC & AF6-120.1 & eBioscience & 0.2 \\
\hline
\end{tabular}

\section{Statistical analyses}

Data are presented as arithmetic means \pm standard deviation. For two-group comparisons, we used Student's $t$-test and for multiple comparisons we used analysis of variance (ANOVA) with Bonferroni posttests after confirming normal distribution with D'Agostino \& Pearson omnibus normality test. Histopathological scores were compared with the Wilcoxon signed-rank test and survival curves with the log-rank/Mantel-Cox test. All data were obtained from independent measurements.

Acknowledgments: We thank S. Keitsch, C. Müller and S. Harde for their excellent help with the animal experiments and D. Herrmann for his excellent technical assistance with the LC-MS/MS analyses. We thank Beat Haenni for the electron microscopical preparations. Electron micrographs were acquired on equipment supported by the Microscopy Imaging Center (MIC) of the University of Bern. 
Funding: This work was supported by DFG grant GU 335-35/1 to EG and GRK 2098 (Funder Id: 10.13039/501100001659) to $\mathrm{KBF}$ and $\mathrm{EG}$.

Conflict of interest statement: The authors declare that they have no conflict of interest.

\section{References}

Alayoubi, A.M., Wang, J.C., Au, B.C., Carpentier, S., Garcia, V., Dworski, S., El-Ghamrasni, S., Kirouac, K.N., Exertier, M.J., Xiong, Z.J., et al. (2013). Systemic ceramide accumulation leads to severe and varied pathological consequences. EMBO Mol. Med. 5, 827-842.

Antonarakis, S.E., Valle, D., Moser, H.W., Moser, A., Qualman, S.J., and Zinkham, W.H. (1984). Phenotypic variability in siblings with Farber disease. J. Pediatr. 104, 406-409.

Bao, X.H., Tian, J.M., Ji, T.Y., and Chang, X.Z. (2017). [A case report of childhood Farber's disease and literature review]. Zhonghua Er Ke Za Zhi. 55, 54-58.

Ben-Yoseph, Y., Gagne, R., Parvathy, M.R., Mitchell, D.A., and Momoi, T. (1989). Leukocyte and plasma N-laurylsphingosine deacylase (ceramidase) in Farber disease. Clin. Genet. 36, 38-42.

Coant, N., Sakamoto, W., Mao, C., and Hannun, Y.A. (2017). Ceramidases, roles in sphingolipid metabolism and in health and disease. Adv. Biol. Regul. 63, 122-131.

Dulaney, J.T., Milunsky, A., Sidbury, J.B., Hobolth, N., and Moser, H.W. (1976). Diagnosis of lipogranulomatosis (Farber disease) by use of cultured fibroblasts. J. Pediatr. 89, 59-61.

Dworski, S., Berger, A., Furlonger, C., Moreau, J.M., Yoshimitsu, M., Trentadue, J., Au, B.C., Paige, C.J., and Medin, J.A. (2015). Markedly perturbed hematopoiesis in acid ceramidase deficient mice. Haematologica 100, e162-e165.

Dworski, S., Lu, P., Khan, A., Maranda, B., Mitchell, J.J., Parini, R., Di Rocco, M., Hugle, B., Yoshimitsu, M., Magnusson, B., et al. (2017). Acid ceramidase deficiency is characterized by a unique plasma cytokine and ceramide profile that is altered by therapy. Biochim. Biophys. Acta 1863, 386-394.

Dyment, D.A., Sell, E., Vanstone, M.R., Smith, A.C., Garandeau, D., Garcia, V., Carpentier, S., Le Trionnaire, E., Sabourdy, F., Beaulieu, C.L., et al. (2014). Evidence for clinical, genetic and biochemical variability in spinal muscular atrophy with progressive myoclonic epilepsy. Clin. Genet. 86, 558-563.

Ehlert, K., Roth, J., Frosch, M., Fehse, N., Zander, N., and Vormoor, J. (2006). Farber's disease without central nervous system involvement: bone-marrow transplantation provides a promising new approach. Ann. Rheum. Dis. 65, 1665-1666.

Eliyahu, E., Park, J.H., Shtraizent, N., He, X., and Schuchman, E.H. $\underline{\text { (2007). Acid ceramidase is a novel factor required for early }}$ embryo survival. FASEB J. 21, 1403-1409.

Farber, S., Cohen, J., and Uzman, L.L. (1957). Lipogranulomatosis, a new lipo-glycoprotein storage disease. J. Mt. Sinai Hosp. N. Y. 24, 816-837.

Fensom, A.H., Benson, P.F., Neville, B.R., Moser, H.W., Moser, A.E., and Dulaney, J.T. (1979). Prenatal diagnosis of Farber's disease. Lancet ii, 990-992.
Filippov, V., Song, M.A., Zhang, K., Vinters, H.V., Tung, S., Kirsch, W.M., Yang, J., and Duerksen-Hughes, P.J. (2012). Increased ceramide in brains with Alzheimer's and other neurodegenerative diseases. J. Alzheimers Dis. 29, 537-547.

Filosto, M., Aureli, M., Castellotti, B., Rinaldi, F., Schiumarini, D., Valsecchi, M., Lualdi, S., Mazzotti, R., Pensato, V., Rota, S., et al. (2016). ASAH1 variant causing a mild SMA phenotype with no myoclonic epilepsy: a clinical, biochemical and molecular study. Eur. J. Hum. Genet. 24, 1578-1583.

Fujiwaki, T., Hamanaka, S., Koga, M., Ishihara, T., Nishikomori, R., Kinoshita, E., and Furusho, K. (1992). A case of Farber disease. Acta Paediatr. Jpn. 34, 72-79.

Fujiwaki, T., Hamanaka, S., Tate, S., Inagaki, F., Suzuki, M., Suzuki, A., and Mori, C. (1995). Tissue accumulation of sulfatide and GM3 ganglioside in a patient with variant Farber disease. Clin. Chim. Acta 234, 23-36.

Gan, J.J., Garcia, V., Tian, J., Tagliati, M., Parisi, J.E., Chung, J.M., Lewis, R., Baloh, R., Levade, T., and Pierson, T.M. (2015). Acid ceramidase deficiency associated with spinal muscular atrophy with progressive myoclonic epilepsy. Neuromuscul. Disord. 25, 959-963.

Gulbins, E., Palmada, M., Reichel, M., Luth, A., Bohmer, C., Amato, D., Müller, C.P., Tischbirek, C.H., Groemer, T.W., Tabatabai, G., et al. (2013). Acid sphingomyelinase-ceramide system mediates effects of antidepressant drugs. Nat. Med. 19 , 934-938.

He, X., Dworski, S., Zhu, C., DeAngelis, V., Solyom, A., Medin, J.A., Simonaro, C.M., and Schuchman, E.H. (2017). Enzyme replacement therapy for Farber disease: proof-of-concept studies in cells and mice. Biochim. Biophys. Acta Clin. 7, 85-96.

Huston, J.P., Kornhuber, J., Mühle, C., Japtok, L., Komorowski, M., Mattern, C., Reichel, M., Gulbins, E., Kleuser, B., Topic, B., et al. (2016). A sphingolipid mechanism for behavioral extinction. J. Neurochem. 137, 589-603.

Iqbal, J., Walsh, M.T., Hammad, S.M., and Hussain, M.M. (2017). Sphingolipids and lipoproteins in health and metabolic disorders. Trends Endocrinol. Metab. 28, 506-518.

Jones, E.E., Dworski, S., Canals, D., Casas, J., Fabrias, G., Schoenling, D., Levade, T., Denlinger, C., Hannun, Y.A., Medin, J.A., et al. (2014). On-tissue localization of ceramides and other sphingolipids by MALDI mass spectrometry imaging. Anal. Chem. 86, 8303-8311.

Kosinska, M.K., Liebisch, G., Lochnit, G., Wilhelm, J., Klein, H., Kaesser, U., Lasczkowski, G., Rickert, M., Schmitz, G., and Steinmeyer, J. (2014). Sphingolipids in human synovial fluid-a lipidomic study. PLoS One 9, e91769.

Kostik, M.M., Chikova, I.A., Avramenko, V.V., Vasyakina, L.I., Le Trionnaire, E., Chasnyk, V.G., and Levade, T. (2013). Farber lipogranulomatosis with predominant joint involvement mimicking juvenile idiopathic arthritis. J. Inherit. Metab. Dis. 36, 1079-1080.

Kudoh, T. and Wenger, D.A. (1982). Diagnosis of metachromatic leukodystrophy, Krabbe disease, and Farber disease after uptake of fatty acid-labeled cerebroside sulfate into cultured skin fibroblasts. J. Clin. Invest. 70, 89-97.

Levade, T., Tempesta, M.C., and Salvayre, R. (1993). The in situ degradation of ceramide, a potential lipid mediator, is not completely impaired in Farber disease. FEBS Lett. 329, 306-312. 
Levade, T., Moser, H.W., Fensom, A.H., Harzer, K., Moser, A.B., and Salvayre, R. (1995). Neurodegenerative course in ceramidase deficiency (Farber disease) correlates with the residual lysosomal ceramide turnover in cultured living patient cells. J. Neurol. Sci. 134, 108-114.

Levade, T., Leruth, M., Graber, D., Moisand, A., Vermeersch, S., Salvayre, R., and Courtoy, P.J. (1996). In situ assay of acid sphingomyelinase and ceramidase based on LDL-mediated lysosomal targeting of ceramide-labeled sphingomyelin. J. Lipid Res. 37, 2525-2538.

Li, C.M., Hong, S.B., Kopal, G., He, X., Linke, T., Hou, W.S., Koch, J., Gatt, S., Sandhoff, K., and Schuchman, E.H. (1998). Cloning and characterization of the full-length CDNA and genomic sequences encoding murine acid ceramidase. Genomics 50 , 267-274.

Li, C.M., Park, J.H., He, X., Levy, B., Chen, F., Arai, K., Adler, D.A., Disteche, C.M., Koch, J., Sandhoff, K., et al. (1999). The human acid ceramidase gene (ASAH): structure, chromosomal location, mutation analysis, and expression. Genomics 62, 223-231.

Li, C.M., Park, J.H., Simonaro, C.M., He, X., Gordon, R.E., Friedman, A.H., Ehleiter, D., Paris, F., Manova, K., Hepbildikler, S., et al. (2002). Insertional mutagenesis of the mouse acid ceramidase gene leads to early embryonic lethality in homozygotes and progressive lipid storage disease in heterozygotes. Genomics 79, 218-224.

Mondal, R.K., Nandi, M., Datta, S., and Hira, M. (2009). Disseminated lipogranulomatosis. Indian Pediatr. 46, 175-177.

Moser, H.W.L.T., Fensom, A. H., Levade, T., Sandhoff, K. (2001). Acid ceramidase deficiency: Farber lipogranulomatosis. In: The Metabolic and Molecular Bases of Inherited Diseases (New York: McGraw-Hill Inc.), pp. 3573-3588.

Peister, A., Mellad, J.A., Larson, B.L., Hall, B.M., Gibson, L.F., and Prockop, D.J. (2004). Adult stem cells from bone marrow (MSCs) isolated from different strains of inbred mice vary in surface epitopes, rates of proliferation, and differentiation potential. Blood 103, 1662-1668.

Quillin, R.C., 3rd, Wilson, G.C., Nojima, H., Freeman, C.M., Wang, J., Schuster, R.M., Blanchard, J.A., Edwards, M.J., Gandhi, C.R., Gulbins, E., et al. (2015). Inhibition of acidic sphingomyelinase reduces established hepatic fibrosis in mice. Hepatol. Res. 45, 305-314.

Schuchman, E.H. and Desnick, R.J. (2017). Types A and B NiemannPick disease. Mol. Genet. Metab. 120, 27-33.

Shtraizent, N., Eliyahu, E., Park, J.H., He, X., Shalgi, R., and Schuchman, E.H. (2008). Autoproteolytic cleavage and activation of human acid ceramidase. J. Biol. Chem. 283, 11253-11259.

Sikora, J., Dworski, S., Jones, E.E., Kamani, M.A., Micsenyi, M.C., Sawada, T., Le Faouder, P., Bertrand-Michel, J., Dupuy, A., Dunn, C.K., et al. (2017). Acid ceramidase deficiency in mice results in a broad range of central nervous system abnormalities. Am. J. Pathol. 187, 864-883.

Singh, I., Pahan, K., Khan, M., and Singh, A.K. (1998). Cytokinemediated induction of ceramide production is redox-sensitive. Implications to proinflammatory cytokine-mediated apoptosis in demyelinating diseases. J. Biol. Chem. 273, 20354-20362.
Sólyom, A., Karabul, N., Hügle, B., Simonaro, C., and Schuchman, E. (2014). Polyarticular arthritis as presenting feature of Farber disease: a lysosomal storage disease involving inflammation. Pediatr. Rheumatol. 12, P285.

Sugita, M., Dulaney, J.T., and Moser, H.W. (1972). Ceramidase deficiency in Farber's disease (lipogranulomatosis). Science 178, 1100-1102.

Sugita, M., Willians, M., Dulaney, J.T., and Moser, H.W. (1975). Ceramidase and ceramide synthesis in human kidney and cerebellum. Description of a new alkaline ceramidase. Biochim. Biophys. Acta 398, 125-131.

Teichgräber, V., Ulrich, M., Endlich, N., Riethmüller, J., Wilker, B., De Oliveira-Munding, C.C., van Heeckeren, A.M., Barr, M.L., von Kürthy, G., et al. (2008). Ceramide accumulation mediates inflammation, cell death and infection susceptibility in cystic fibrosis. Nat. Med. 14, 382-391.

Torcoletti, M., Petaccia, A., Pinto, R.M., Hladnik, U., Locatelli, F., Agostoni, C., and Corona, F. (2014). Farber disease in infancy resembling juvenile idiopathic arthritis: identification of two new mutations and a good early response to allogeneic haematopoietic stem cell transplantation. Rheumatology (Oxford) 53, 1533-1534.

Tsuboi, K., Sun, Y.X., Okamoto, Y., Araki, N., Tonai, T., and Ueda, N. (2005). Molecular characterization of $\mathrm{N}$-acylethanolaminehydrolyzing acid amidase, a novel member of the choloylglycine hydrolase family with structural and functional similarity to acid ceramidase. J. Biol. Chem. 280, 1108211092.

Vasiliauskaite-Brooks, I., Sounier, R., Rochaix, P., Bellot, G., Fortier, M., Hoh, F., De Colibus, L., Bechara, C., Saied, E.M., Arenz, C., et al. (2017). Structural insights into adiponectin receptors suggest ceramidase activity. Nature 544, 120-123.

Yeager, A.M., Uhas, K.A., Coles, C.D., Davis, P.C., Krause, W.L., and Moser, H.W. (2000). Bone marrow transplantation for infantile ceramidase deficiency (Farber disease). Bone Marrow Transplant. 26, 357-363.

Yu, F.P., Islam, D., Sikora, J., Dworski, S., Gurka, J., Lopez-Vasquez, L., Liu, M., Kuebler, W.M., Levade, T., Zhang, H., et al. (2018). Chronic lung injury and impaired pulmonary function in a mouse model of acid ceramidase deficiency. Am. J. Physiol. Lung Cell. Mol. Physiol. 314, L406-L420.

Zaugg, P., Djonov, V., Fuchtbauer, E.M., and Draeger, A. (1999). Sorting of murine vascular smooth muscle cells during wound healing in the chicken chorioallantoic membrane. Exp. Cell. Res. 253, 599-606.

Zhang, Z., Mandal, A.K., Mital, A., Popescu, N., Zimonjic, D., Moser, A., Moser, H., and Mukherjee, A.B. (2000). Human acid ceramidase gene: novel mutations in Farber disease. Mol. Genet. Metab. 70, 301-309.

Zhou, J., Tawk, M., Tiziano, F.D., Veillet, J., Bayes, M., Nolent, F., Garcia, V., Servidei, S., Bertini, E., Castro-Giner, F., et al. (2012). Spinal muscular atrophy associated with progressive myoclonic epilepsy is caused by mutations in ASAH1. Am. J. Hum. Genet. 91, 5-14.

Supplementary Material: The online version of this article offers supplementary material (https://doi.org/10.1515/hsz-2018-0170). 